\title{
1 TITLE: Post-capillary venules is the locus for transcytosis of 2 therapeutic nanoparticles to the brain
}

3

\section{Authors:}

5 Krzysztof Kucharz*\#1, Kasper Kristensen*2,3, Kasper Bendix Johnsen ${ }^{2,3}$, Mette 6 Aagaard Lund ${ }^{2,3}$, Micael Lønstrup ${ }^{1}$, Torben Moos ${ }^{4}$, Thomas Lars Andresen ${ }^{2,3}$, Martin 7 Johannes Lauritzen ${ }^{\# 1,5}$.

\section{Affiliations}

$10 \quad{ }^{1}$ Department of Neuroscience, University of Copenhagen, Copenhagen, Denmark

$11{ }^{2}$ Department of Health Technology, Technical University of Denmark, Kgs. Lyngby, Denmark

$12{ }^{3}$ Center for Nanomedicine and Theranostics, Technical University of Denmark, Kgs. Lyngby,

13 Denmark

$14 \quad{ }^{4}$ Department of Health Science and Technology, Aalborg University, Denmark

$15{ }^{5}$ Department of Clinical Neurophysiology, Rigshospitalet-Glostrup, Copenhagen, Denmark

17 Author list footnotes

$18 *$ - these authors contributed equally.

\# - corresponding authors: K.K. (kucharz@ @und.ku.dk) and M. J. L. (

\section{HIGHLIGHTS:}

- Integration of drug carrier nanotechnology with two-photon microscopy in vivo

- Real-time nanoscale-resolution imaging of nanoparticle transcytosis to the brain

34 - Distinct trafficking pattern in the endothelium of cerebral venules and capillaries

35 - Venules, not capillaries, is the locus for brain uptake of therapeutic nanoparticles 


\section{SUMMARY:}

2

3 Treatments of neurodegenerative diseases require biologic drugs to be actively

4 transported across the blood-brain barrier (BBB). To answer outstanding questions

5 regarding transport mechanisms, we determined how and where transcytosis occurs at

6 the BBB. Using two-photon microscopy, we characterized the transport of therapeutic

7 nanoparticles at all steps of delivery to the brain and at the nanoscale resolution in

8 vivo. Transferrin receptor-targeted nanoparticles were taken up by endothelium at

9 capillaries and venules, but not at arterioles. The nanoparticles moved unobstructed

10 within endothelial cells, but transcytosis across the BBB occurred only at post-

11 capillary venules, where endothelial and glial basement membranes form a

12 perivascular space that can accommodate biologics. In comparison, transcytosis was

13 absent in capillaries with closely apposed basement membranes. Thus, post-capillary

14 venules, not capillaries, provide an entry point for transport of large molecules across

15 the BBB, and targeting therapeutic agents to this locus may be an effective way for

16 treating brain disorders.

17

\section{KEYWORDS:}

Blood-brain barrier, transcytosis, two-photon imaging, nanoparticle, transferrin receptor, in vivo, awake mice, drug delivery, capillaries, venules.

\section{INTRODUCTION}

26 The blood-brain barrier (BBB) is impermeable to most blood-borne substances 27 (Abbott et al., 2010). The paracellular route across the BBB is limited by junctional complexes between brain endothelial cells (BEC). Diffusion across BEC is possible, but restricted to low-molecular weight hydrophobic compounds, and most lipophilic drugs show negligible brain uptake because of the rapid outward transport of xenobiotics by BEC efflux pumps (Mollgard et al., 2017; Pardridge, 2012). Large molecules can enter the brain, but only via vesicular transport, i.e. transcytosis.

33 However, this route is highly selective and strongly suppressed by recently identified 34 homeostatic mechanisms (Ben-Zvi et al., 2014; De Bock et al., 2016; Janiurek et al., 
1 2019; Tietz and Engelhardt, 2015). Consequently, while the low BBB permeability 2 protects the brain against circulating pathogens, it also precludes more than $98 \%$ of 3 neuroprotective compounds from reaching the brain, rendering central nervous system 4 (CNS) disorders resistant to most conventional therapies (Banks, 2016; Pardridge, 5 2002, 2012).

6 Drug delivery systems that adapted the receptor-mediated transcytosis (RMT) to 7 shuttle large therapeutic cargo across the BBB are currently at the forefront of the 8 modern pharmacology (Lajoie and Shusta, 2015; Pardridge, 2020; Pulgar, 2018).

9 Coupling carrier molecules to RMT ligands may enhance delivery of therapeutics to 10 the brain, and the flagship RMT ferrying receptor in the brain is the transferrin 11 receptor (TfR) (Johnsen et al., 2019b; Pardridge, 2002, 2020). Nanoparticles, such as 12 liposomes, are versatile drug carriers that can encapsulate a variety of xenobiotics, 13 and functionalized with TfR ligands represent a promising drug delivery approach 14 with diverse therapeutic opportunities (Johnsen et al., 2019b; Pardridge, 2020). The 15 translational potential of TfR-targeted nanoparticles has been tested in preclinical 16 models of Parkinson's, Alzheimer's, Huntington's disease, brain cancer, and stroke, 17 but the level of drug delivery levels rarely meet the thresholds for clinical significance 18 due to failure in nanoparticle transcytosis (Johnsen et al., 2019b).

19 To address this issue, we examined how cerebral vessels handle clinically relevant nanoparticles in the living, intact brain. We employed two-photon microscopy in anesthetized and awake mice to characterize the pharmacokinetics of TfR-targeted nanoparticles at the BBB, intracellular trafficking patterns in BECs, and transcytosis into the brain parenchyma using real-time in vivo imaging at nanoscale resolution. We report that TfR-targeted nanoparticles bind efficiently to BECs in venules and capillaries, but are absent in arterioles. Despite the highest uptake by BECs in capillaries, we found that transcytosis occurs only in post-capillary venules that, in contrast to capillaries, are surrounded by a large perivascular space that can accommodate nanoparticle-sized elements (Engelhardt et al., 2017). Our observations indicate that post-capillary venules mediate brain uptake of large drug carriers, which should be considered in the design of next-generation nanoparticles for the treatment of brain disorders. 


\section{RESULTS}

2

3 Two-photon imaging of BBB-targeted nanoparticles in vivo.

4 To investigate how drug nanocarriers interact with the BBB in vivo, we used two-

5 photon fluorescence microscopy in mice. Following microsurgery, the brain was

6 imaged via a cranial window over the somatosensory cortex (Figures 1A and 1B). The

7 nanoparticles were designed to resemble clinically approved nanoliposome

8 formulations (Barenholz, 2012), consisting of a

9 distearoylphosphatidylcholine(DSPC)/cholesterol lipid bilayer surrounding an

10 aqueous lumen and with a PEGylated surface to ensure stability in the blood (Figure

11 1C, Table S1). To enable targeting to the brain via TfR, we coupled the nanoparticles

12 to immunoglobulin G (IgG) monoclonal anti-TfR antibody clone RI7217 (Lee et al.,

13 2000). Prior to two-photon imaging experiments, we validated the nanoparticle TfR

14 targeting system by encapsulating BBB-impermeable cisplatin into nanoparticles

15 (Table S1), and injecting the nanoparticles into the bloodstream. The traces of

16 cisplatin were detected in the brain only for nanoparticles functionalized with RI7217,

17 but not stealth (no targeting antibody), or isotype IgG (no TfR recognition) antibodies

18 (Figure 1D).

19 To enable detection in subsequent two-photon imaging experiments, we fluorescently

20 labeled the nanoparticle lipid bilayer with either Atto488 or Atto550 (Figure 1C).

21 Both the Atto550- and Atto488-labeled nanoparticles (RI7-L-A550 and RI7-L-A488)

22 were administered by single bolus injection into the bloodstream $\left(70 \mathrm{nmol}_{\text {lipid }} / \mathrm{g}_{\text {animal }}\right)$

23 and were subsequently imaged in the brain volume containing pial arterioles and

24 venules, penetrating arterioles, ascending venules, pre- and post-capillary vessels, and

25 capillaries (Figure 1E). The blood-circulating nanoparticles exhibited high

26 fluorescence stability over time, indicating a low filtration rate by peripheral organs

27 (Figures $1 \mathrm{~F}$ and $1 \mathrm{G}$, Movie S1). Nanoparticles retained their single-particle

28 appearance without agglomeration or fusion, as ascertained by laser-extravasation of

29 nanoparticles from the bloodstream into the brain parenchyma after $3 \mathrm{~h}$ in circulation

30 (Figures 1H and 1I). A small fraction of nanoparticles was sequestered by leukocytes,

31 but without adverse effects on the brain and systemic physiology that could

32 compromise the BBB (Figure S1, Movie S2).

33 Nanoparticles in the brain in vivo exhibited properties of a point source signal with a

34 Gaussian distribution peak denoting the center location of the nanoparticle. The 
1 fluorescence distribution standard deviation ( $\sigma$ ) was $0.290 \pm 0.006 \mu \mathrm{m}$ and $2 \quad 0.296 \pm 0.008 \mu \mathrm{m}$ for RI7-L-A550 and RI7-L-A488, respectively, and did not differ 3 between the two distinctively labeled nanoparticle types $(n=20, p=0.5910$, t-test) 4 (Figure 1J). In subsequent experiments, the nanoparticles were considered spatially 5 separated when their peak of fluorescence exceeded $2 \sigma$ from other sources of 6 fluorescence, i.e., vessel lumen, endothelium, or other nanoparticles.

7 Thus, the nanoparticles fulfilled all necessary requirements for in vivo imaging: 8 efficient two-photon excitation and emission of fluorescence with the ability to 9 resolve single nanoparticles, high structural stability in the circulation, and no 10 detrimental effects on the brain and systemic physiology. Targeted nanoparticles selectively associate to capillaries and venules.

13 To delineate vessel lumen, we used FITC-dextran (FITC-dx) injected subsequently to 14 the nanoparticles. The blood-circulating nanoparticles were rapidly recruited to the 15 endothelium, and at $2 \mathrm{~h}$ post-injection, appeared as numerous punctae at vessel walls 16 of the capillaries, and of the pial (Figure 2A), ascending, and post-capillary venules 17 (Figures 2B-2E), but were absent from the arterial branches of the brain 18 microvasculature (Figure $2 \mathrm{~F}$ ). The association was driven by interactions of the TfR$19 \mathrm{Ab}$ with the TfR at the BEC surface and not by stalling of nanoparticles in the vessel lumen, as neither antibody-lacking stealth nanoparticles (Sth-L-A550)(Figures S2A and S2B) nor isotype IgG-functionalized nanoparticles that lack TfR recognition (IgG-L-A550) associated to BECs (Figures S2C and S2D). The nanoparticle association was independent of the type of fluorescent tag, as RI7-L-A488 exhibited the same targeting properties as RI7-L-A550 (Figures S3A-E).

25 Lastly, mice co-injected with both nanoparticles exhibited distinct labeling at the 26 vessel walls from both RI7-L-A488 and RI7-L-A550, attesting that the observed 27 punctae represented single nanoparticles (Figure 2G). Scarce presence of merged signals was attributed to overlapping fluorescence of nanoparticles separated by a distance smaller than the diffraction limit of the microscope (Figure $2 \mathrm{H}$ ).

\section{Vessel type determines association density and kinetics.}

32 We isolated BEC-associated from blood-circulating nanoparticles by excluding the 33 signal of circulating nanoparticles that colocalized with FITC-dx in the vessel lumen 34 (Figure 3A). Three-dimensional (3D) reconstruction revealed a spatially 
1 heterogeneous association of nanoparticles across the vascular tree (Figure 3B). At 2

$2 \mathrm{~h}$ post-injection, the highest density, i.e., number of nanoparticles at the vessel

3 surface, was at BECs of capillaries and venules with an exponential decline in

4 ascending and pial venules (Figure 3D). This observation was consistent with the

5 kinetics of association being fastest in capillaries and slowest in pial venules (Figures

6 3E, S3F, and S3G, Movie S3). Of note, nanoparticle binding was ongoing even after 2

7 h post-injection, indicating that the cellular pool of available TfRs was not saturated

8 at this time (Figure 3E).

9

10 Most of associated nanoparticles are internalized by endothelium.

11 To determine the type of interaction between nanoparticles and BEC, i.e. the ratio 12 between internalized and adhering nanoparticles, we used transgenic mice expressing 13 cytosolic green fluorescent protein (GFP) in the endothelium (Tie2-GFP; see 14 Methods). This allowed for a comprehensive assessment of endothelium morphology 15 with unprecedented details in the living brain (Figures 4A and S4). We could discern 16 the distinct patterns of nuclei and endothelial cell contact sites (Figure 4B), and the 17 fluorescence signal was compatible with imaging of the nanoparticles (Figure 4C).

18 At $2 \mathrm{~h}$ post-injection, TfR-coated nanoparticles exhibited a non-uniform distribution 19 in relation to the vessel wall, appearing either to be internalized or to adhere to the 20 luminal surface of the endothelium (Figure 4D). For each analyzed vessel, we aligned 21 the imaging plane with the center of the vessel radial symmetry (the broadest vessel 22 cross-section), and for each nanoparticle, we extracted the fluorescence profile plot 23 with corresponding endothelium signal (Figure 4E). As a measure of separation, we 24 used the distance between peaks $(\Delta \mathrm{p})$. The precise spatial localization of nanoparticles 25 is non-trivial because of the diffraction limit, therefore we defined the boundary 26 conditions characterizing nanoparticle location using the previously calculated 27 standard deviation $(\sigma)$ of Gaussian fluorescence distribution $(\sigma=0.29 \mu \mathrm{m}$; Figure $1 \mathrm{~J})$.

28 Using conservative inclusion criteria, the nanoparticles were categorized as 29 internalized when separated from endothelium peak signal only by $|\Delta \mathrm{p}|<\sigma$ (high 30 fluorescence overlap) and as adhering when separated from the endothelium peak 31 signal by at least $2 \sigma$, i.e., $|\Delta \mathrm{p}|>2 \sigma$ (minimal fluorescence overlap). The third group 32 consisted of nanoparticles that could not be categorically classified into adhering or 33 internalized and represented the intermediate group (i.e., unresolved, $\sigma \leq|\Delta \mathrm{p}| \leq 2 \sigma$ ).

34 Our boundary conditions were further attested by visual inspection of nanoparticles 
1 (Figure 4F). Given a wide signal point spread function along the Z-axis (depth), we

2 did not analyze capillaries because of their small diameter and high vessel curvature.

3 At $2 \mathrm{~h}$ post-injection, the internalized fractions were $55.9 \%, 48.0 \%$, and $46.3 \%$ of

4 total nanoparticles at the endothelium for main pial venules, pial venules, and

5 ascending venules, respectively (Figure 4G). The corresponding adhering fraction of

6 nanoparticles was $24.0 \%, 30.9 \%$, and $32.8 \%$, while the unresolved fraction was

$720.1 \%, 21.1 \%$, and $20.8 \%$. This indicates that, on average, at least half of the

8 nanoparticles recruited to vessel walls were internalized by endothelium. Noteworthy,

9 within the unresolved fraction, we detected nanoparticles with $\Delta \mathrm{p}<-\sigma$, with

10 prevalences of $7.1 \%, 5.2 \%$, and $3.8 \%$ for main pial venules, pial venules, and

11 ascending venules, respectively. These nanoparticles were located in proximity to the

12 basolateral membrane of BEC, and their prevalence increased with venule diameter.

\section{Directional movement of nanoparticles inside endothelium}

15 Once internalized, the nanoparticles exhibited a relatively high degree of movement 16 over short distances (Figure 5A, Movie S4). The nanoparticles were tracked at 2-3 h 17 post-injection with respect to large vessels, i.e. pial venules, and small vessels, i.e. 18 post-capillary venules with capillaries (Movie S5). We omitted ascending venules 19 because of their varying angled orientation to the imaging plane. From each analyzed vessel, we selected 10 nanoparticles that remained in the imaging plane throughout 30 mins of continuous imaging (time interval between frames=30 s) (Figure 5B). The traces were aligned to the point of origin (Figure 5C), and to avoid underestimation of movement in planar $(x, y)$ coordinates because of vessel curvature, we projected each trace to a vector $(v)$ aligned with a vessel symmetry axis and blood flow direction, 25 both independent from circumference curvature (Figure 5D).

26 The total distance traveled along the vessel axis did not differ between nanoparticles 27 in large venules, and post-capillary venules with capillaries ( $\mathrm{p}=0.1672$, Mann28 Whitney; Figure 5E). Similarly, the end-position of the nanoparticle relative to the origin was the same for all vessel types ( $\mathrm{p}=0.6694$, Mann-Whitney)(Figure 5F). The movement did not occur preferentially along or against the blood flow, as in all vessel

31 types, the median displacement relative to the blood flow direction approximated 0 32 (Figure 5G). Of note, nanoparticles exhibited motility even in capillaries with stalled 33 blood flow (Movie S6). Thus, neither cell morphology, nor differences in blood flow 
1 velocities (Santisakultarm et al., 2012), nor blood flow direction influenced the

2 intracellular movement of the nanoparticles.

3 To determine whether intracellular trafficking of nanoparticles occurs by random

4 movement (e.g., diffusion) or is directional, we performed mean squared

5 displacement (MSD) analysis (see Methods). MSD analysis was performed along the

6 vessel axis $v($ Figure $5 \mathrm{H})$ and $\operatorname{MSD}(\mathrm{t})$ significantly departed from linearity $(\mathrm{p}<0.001$,

$7 \mathrm{n}=673$ nanoparticles, Wald-Wolfowitz runs test). This indicates that the movement in

8 the endothelium contains a significant directional component. This suggested that the

9 nanoparticles might be directed towards specific cell regions, which we assessed in

10 the subsequent experiment.

Nanoparticles distribute to endothelial cell perinuclear area in venules but not in 13 capillaries.

14 Upon internalization, the nanoparticles localized over time to the perinuclear region

15 of endothelium cells in venules, but not in capillaries (Figures 5I and 5J). To quantify

16 the spatial distribution of nanoparticles in venules, we measured the distances from

17 the geometric center of the nucleus to the nanoparticles (Figure 5K). Our kernel

18 density map shows that the highest probability for finding a nanoparticle in venules

19 was in proximity to the nucleus (Figure 5L), at distances of $0.5-2.5 \mu \mathrm{m}$ from the

20 nucleus boundary and with numbers decreasing at intermediate $(2.5-5 \mu \mathrm{m})$ and distal

$21(>5 \mu \mathrm{m})$ regions (Figure 5M). Of note, neither vessel type exhibited clustering of

22 nanoparticles at the endothelium perimeter (Figure $5 \mathrm{~N}$ ). This indicated that

23 nanoparticles do not stall in the cytosol areas that contain dense cytoskeleton elements

24 that support cell contact sites, and that nanoparticles do not wedge between adjacent

25 endothelial cells.

27 Nanoparticles are transcytosed to the brain, but only at venules.

28 The possibility of large nanoparticle transcytosis across the BBB is disputed (Clark 29 and Davis, 2015; Lindqvist et al., 2016; van Rooy et al., 2011; Wiley et al., 2013).

30 Here, we detected nanoparticles present at the basolateral side of the endothelium in 31 both capillaries and venules (Figure 6A). This could result from lateral displacement 32 of nanoparticles, i.e. movement within endothelium along the vessel (Figure 6B). 33 However, in contrast to capillaries, nanoparticles in venules exhibited translocation 34 normal to the vessel lumen (Figure 6C). We observed that nanoparticles that 
1 associated with the vessel wall slowed down, but once transcytosed, they rapidly 2 accelerated into the perivascular space (Figure 6D, Movie S7). This occurred only in 3 pial and post-capillary venules and not in capillaries, indicating that the vessel type 4 crucially determines successful transcytosis. Of note, we detected rare events in which 5 blood-borne cells that sequestered nanoparticles crossed the BEC in venules (Movie 6 S8), consistent with previous studies of immune-cell trafficking in the brain 7 (Engelhardt et al., 2017).

8 Continuous high-frequency time-lapse imaging required to capture transcytosis events 9 may potentially damage the $\mathrm{BBB}$, leading to extravasation of the blood-borne components to the brain (Choi et al., 2011). Given this possibility, along with the rarity of transcytosis events and limited span of acute imaging experiments ( 4 h), we next performed long-term two-photon imaging on awake and mobile mice with 13 chronic cranial window implants (Figures 6E and 6F; see Methods). We imaged the 14 brains of awake Tie2-GFP mice 10-day post-surgery, to identify the cortical areas that contained large venules (Figure 6G). We then reassessed the same loci 2 days later, and subsequently, injected RI7-L-A550 into the bloodstream $(50 \mu \mathrm{L} 70$ $\left.17 \mathrm{nmol}_{\text {lipid }} / \mathrm{g}_{\text {animal }}\right)$. Next, we imaged the animals again (30 min post-injection) to ensure 18 that the endothelium remained structurally intact and examined the same region at 1 and 2 days post-injection (Figure 6G). Already at 1 day post-injection, the animals exhibited no significant presence of nanoparticles in the circulation, indicating effective clearance of RI7-L-A550 from the bloodstream. However, at this timepoint we detected substantial amounts of nanoparticles on the abluminal side of the endothelium in the brain parenchyma surrounding venules. This fraction of nanoparticles significantly decreased between 1 and 2 days post-injection but was still abundant, indicating high retention (Figure 6G and H). In contrast to pial, ascending, and post-capillary venules, nanoparticles failed to transcytose to the brain tissue close to capillaries and the arterial branches of the microvasculature (Figure 6G). This confirmed that transcytosis of nanoparticles across the BBB occurs at venules and not at other segments of the brain microvasculature.

\section{DISCUSSION}

34 Understanding the pharmacokinetics of drug-carrying nanoparticles at the BBB in 
1 vivo is crucial for future drug delivery strategies. For nearly two decades, drug 2 delivery studies have faced methodological challenges to determine how 3 nanoparticles may penetrate the BBB. Our current knowledge on nanoparticle-based 4 drug delivery across the BBB is deduced from chemically processed tissue and 5 biodistribution studies, or whole-brain imaging techniques (Cabezon et al., 2015; 6 Johnsen et al., 2019b; Pardridge, 2012). Moreover, the microanatomy of brain tissue 7 surrounding microvessels vastly differs among arterioles, capillaries, and venules 8 (Engelhardt et al., 2017), but how this heterogeneity of CNS vascular anatomy defines 9 distinct BBB compartments is unclear. Here, using two-photon microscopy, we 10 provide direct insight into nanoparticle delivery through all stages of endothelial 11 vesicular transport: binding, uptake, intracellular trafficking, and exocytosis in the 12 living brain. We report that clinically relevant formulations of TfR-targeted 13 nanoparticles rapidly associate with the BECs, with preferential distribution to 14 capillaries. Upon internalization, nanoparticles exhibit movement kinetics 15 independent of the vessel type, and with subcellular distribution profiles that differ 16 between venules and capillaries. Despite the highest density of nanoparticles in 17 capillaries, transcytosis across the BBB in vivo occurs only in venules (Figure 7), the 18 same vascular segment that is involved in immune-cell trafficking (Engelhardt et al., 19 2017).

20 The association of nanoparticles to the endothelium rapidly declined from capillaries 21 to venules, inversely proportional to the vessel diameter and consistent with the TfR 22 expression profile (Ge et al., 2005; Jefferies et al., 1984). TfR is a recycling receptor 23 with $\sim 10 \%$ of the total receptor pool present at the surface of BECs (van Gelder et al., 24 1995). This amount was sufficient to maintain steady and ongoing recruitment of 25 TfR-targeted nanoparticles to the vessel wall without saturation of the receptor pool, 26 even at the high densities in capillaries. Although anti-TfR antibodies readily cross 27 the BBB (Johnsen et al., 2019b; Niewoehner et al., 2014; Yu et al., 2011), there is a 28 fundamental disagreement as to whether significantly larger TfR-targeted 29 nanoparticles actually enter the brain (Clark and Davis, 2015; Lindqvist et al., 2016; 30 van Rooy et al., 2011; Wiley et al., 2013). Here, we provide direct evidence of TfR31 mediated transcytosis of nanoparticles across the endothelial cells of the post32 capillary venules, and challenge the concept that nanoparticles undergo transport 33 across the BBB selectively at capillary endothelial cells. 
1 The key question is why the highest density of associated nanoparticles in capillaries

2 did not translate to the vascular segment of transcytosis. The lack of nanoparticle

3 passage in capillaries can not be explained solely by impaired dissociation of

4 antibody-functionalized nanoparticle from TfR during exocytosis (Couch et al., 2013;

5 Yu et al., 2011), as transcytosis would also be absent at venules. Instead, we propose

6 that the microanatomy of the tissue surrounding microvessels determines successful

7 nanoparticle entry into the brain, specifically the size of the perivascular space and

8 organization of the basement membrane. Large and post-capillary venules are

9 surrounded by perivascular space located between the endothelium basement

10 membrane and astrocyte glia limitans, but in contrast to venules, capillaries are

11 devoid of perivascular space because both membranes are fused (Engelhardt et al.,

12 2017; Thomsen et al., 2017). This principal anatomical brain feature is present in both

13 murine and human brains (Zhang et al., 1990).

14 We propose that nanoparticles are more likely to transcytose via the route of the least 15 resistance, i.e. into the perivascular space of venules; instead of entering the more 16 restrictive compartment, i.e. the brain parenchyma at the capillary segment (Foley et 17 al., 2012; Wardlaw et al., 2020). By analogy, translocation of blood-borne leukocytes 18 across the BEC into the brain is also possible only at the microvascular segments 19 surrounded by perivascular space capable of accommodating the cells, i.e. at post20 capillary venules (Engelhardt et al., 2017). It also is unlikely that nanoparticles were 21 transcytosed at capillaries and subsequently relocated downstream along the capillary 22 wall into the perivascular space of venules due to the interstitial fluid flow. First, the 23 hydraulic resistance around the capillaries is too high for interstitial fluid flow 24 (Faghih and Sharp, 2018). Second, we detected no significant nanoparticle movement 25 following laser extravasation to the brain tissue surrounding capillaries, compared to 26 the rapid progression of nanoparticles extravasated from venules into the perivascular 27 space (Movie S9). Third, if transcytosis occurred in capillaries, a significant fraction 28 of nanoparticles of $>100 \mathrm{~nm}$ diameter would likely become trapped in the protein 29 meshwork of fused capillary basement membranes (Cabezon et al., 2015; Muldoon et 30 al., 1999), but this we did not observe in chronic imaging experiments.

31 The major concern of the large nanoparticle-based drug delivery systems is the ability 32 of transcytosed nanovehicles to progress within the extracellular space (ECS). In vivo 
1 diffusion experiments estimate the ECS to be in the range of 38-64 $\mathrm{nm}$ (Thorne and

2 Nicholson, 2006), although recent super-resolution imaging revealed ECS clefts of

$3 \sim 100 \mathrm{~nm}$ (Tonnesen et al., 2018). These dimensions may not restrict the movement of,

4 e.g., antibodies, but may exclude significantly larger nanoparticles from entry and

5 progression within the ECS (Nance et al., 2012; Thorne and Nicholson, 2006). Here,

6 most nanoparticles were detected in perivascular areas, even after 2 days post-

7 injection, which indicates high perivascular retention. However, we also observed

8 nanoparticles at distances from post-capillary venules corresponding to the location of

9 neuropil, indicating successful passage into the ECS. How 130-nm nanoparticles

10 might travel within the ECS is unclear but appears to be highly dependent on the level

11 of PEGylation (Nance et al., 2012). In the murine brain, neurons are located on

12 average within short $(\sim 15 \mu \mathrm{m})$ distances from brain capillaries (Tsai et al., 2009).

13 Although TfR may potentially act as an intraparenchymal target for neurons (Chen

14 and Liu, 2012; Farias et al., 2015), we observed no cellular association patterns of the

15 nanoparticles suggestive of binding to TfRs, which are enriched at the neuronal

16 somata (Farias et al., 2015).

17 In contrast to microvessels embedded in the brain parenchyma, the vasculature in the

18 dura lacks the BBB (Engelhardt et al., 2017; Weller et al., 2018). Contrary to a recent

19 study (Lam et al., 2018), we detected no TfR-targeted nanoparticles in the dura.

20 However, we did observe blood-borne immune cells that infiltrated the brain, carrying

21 along nanoparticles that were hijacked from the bloodstream. Thus, nanoparticles

22 could also enter the CNS in a manner independent of vesicular transcytosis. This

23 phenomenon, in a similar manner to transcytosis, also occurred exclusively in

24 venules. The leakage of large blood-borne macromolecules can reportedly occur via

25 wedging of leukocytes between endothelial cells in venules but not in capillaries

26 (Claudio et al., 1990). However, this phenomenon is associated with severe

27 autoimmune reactions, and we did not detect the clustering of nanoparticles at BEC

28 contact sites.

29 An important consideration is to what extent brain microanatomy might influence 30 nanoparticle transcytosis compared to other BEC features along the vascular 31 segments. First, colloidal glycocalyx at the BEC luminal side may potentially bury 32 macromolecules within its matrix or repel negatively charged nanoconstructs, 33 impairing endocytosis (Cheng et al., 2016; Gromnicova et al., 2016). Indeed, we 
1 detected that the internalization of nanoparticles was not an all-or-nothing 2 phenomenon, and $\sim 30-40 \%$ total nanoparticles recruited from the blood circulation 3 were restricted to the luminal side of BEC. Although enzymatic shedding of 4 glycocalyx does not improve BEC uptake of positively charged nanoparticles 5 (Gromnicova et al., 2016), we cannot exclude that glycocalyx might affect slightly 6 negatively charged particles (Kutuzov et al., 2018), and in a vessel-type-dependent 7 manner.

8 Second, our data show that after internalization, the nanoparticles exhibited similar 9 movement dynamics regardless of vessel type and consistent with cytoskeleton10 assisted transcytosis (Soldati and Schliwa, 2006). However, in contrast to capillaries, 11 post-capillary and pial venules exhibited the preferential distribution of nanoparticles 12 to perinuclear areas, which corresponds to the position of late endosomes in the 13 transcytotic pathway that contain components directed for degradation (De Bock et 14 al., 2016; Grant and Donaldson, 2009). Although this suggests a more restrictive 15 intracellular barrier for the trafficking of nanoparticles in venules than in capillaries, it 16 supports the notion that despite segmental differences in RMT, it is ultimately the 17 microanatomy of the brain surrounding microvessels that plays a decisive role for successful transcytosis.

Lastly, the vessel-dependent manner in which the brain handles TfR-targeted nanoparticles may potentially explain conflicting reports on the delivery of other TfRtargeted entities, such as antibodies, where the robust ability to penetrate the BBB is often opposed by evidence for the same type of entity indefinitely trapped within the capillaries (Friden et al., 1991; Moos and Morgan, 2001). Our findings may be of relevance for other than TfR-targeting approaches and potentially explain why other targeting ligands e.g. COG133, angiopep-2, or CRM197 that performed successfully 27 in capillaries in vitro, failed to enhance nanoliposome uptake in vivo (van Rooy et al., 2011). Our methodological platform is first to describe the nanoparticle fate in the living brain at nanoscale resolution in live, and also, awake animals. It is suitable for examining the potential effects of disease states on large molecule therapeutics delivery, e.g. during stroke-induced imbalance in transcytosis (Knowland et al., 2014), reduction of perivascular spaces (Mestre et al., 2018), or ECS changes in edema or during brain activity (Tonnesen et al., 2018). Our findings may also help to 34 avoid pitfalls in design of drug delivery systems, e.g. in Alzheimer disease, where A $\beta$ 
1 is deposited along pericapillary and periarteriolar spaces (Tarasoff-Conway et al.,

2 2015), rather than in venular perivascular space, which is the locus of nanoparticle

3 transcytosis.

4 In summary, we reveal the transcytosis pathway across the BBB of targeted clinically

5 relevant nanoparticles. Our identification of post-capillary venules as the key site for

6 transcytosis may aid efforts to develop efficient therapeutic approaches for drug

7 delivery to the CNS. As an accessible part of the vascular tree, post-capillary venules

8 may be poised to serve as a preferred route for macromolecule and nanoparticle

9 delivery across the BBB.

10

11 ACKNOWLEDGMENTS

12

13 This study was supported by Lundbeck Foundation Research Initiative on Brain 14 Barriers and Drug Delivery (\#R392-2018-2266); Det Frie Forskningsråd (\#060215 01965B); Nordea Foundation Grant to the Center for Healthy Aging (\#114995). We 16 thank Nikolay Kutuzov for feedback on data analysis, Serhii Kostrikov for his 17 assistance in cisplatin experiments, and Kjeld Møllgård for his comments on the 18 manuscript.

\section{AUTHOR CONTRIBUTIONS (CRediT taxonomy).}

22 Conceptualization, Kr. Ku., Ka. Kr. and M. J. L.; Methodology, Kr. Ku. and Ka. Kr.; 23 Formal Analysis, Kr. Ku.; Investigation, Kr. Ku., Ka. Kr., K.B.J., M. A. L., and M. 24 L.; Resources - T.M., T. L. A. and M. J. L.; Writing - Original Draft, Kr. Ku.; and M. 25 J. L.; Writing - Review \& Editing, Kr. Ku., Ka. Kr., K.B.J., T.M., and M.J.L.; 26 Visualization, Kr. Ku.; Funding Acquisition, T.M., T. L. A. and M. J. L.; Supervision 27 M. J. L.

\section{DECLARATION OF INTERESTS}

All authors declare no conflict of interest. 


\section{Methods}

3

\section{Animals}

5 All animal experiments were approved by the Danish National Ethics Committee and

6 followed ARRIVE guidelines. We used wild-type C57B1/6 mice, age 5-7 months

7 (23-31 g) and age-matched (25-32 g) homozygous $\mathrm{Tg}$ (TIE2GFP)287Sato/J

8 transgenic reporter mice (Tie2-GFP mice, \#003658, The Jackson Laboratory)

9 expressing GFP under the endothelial-specific receptor tyrosine kinase (Tie2)

10 promoter (Motoike et al., 2000).

11

12 Animal preparation for acute imaging

13 Surgery was performed as previously described, with minor modifications (Kucharz 14 and Lauritzen, 2018). Briefly, animals were anesthetized by intraperitoneal (i.p.) 15 injection of xylazine $\left(10 \mu \mathrm{g} / \mathrm{g}_{\text {animal }}\right)$ and ketamine $\left(60 \mu \mathrm{g} / \mathrm{g}\right.$, then $30 \mu \mathrm{g} / \mathrm{g}_{\text {animal }}$, at 20 $1625 \mathrm{~min}$ intervals). A tracheotomy was performed for mechanical respiration (180-220 $17 \mu \mathrm{L}$ volume at 190-240 strokes/min; MiniVent Type 845, Harvard Apparatus) with $18 \mathrm{O}_{2}$-supplemented air $(1.5-2 \mathrm{~mL} / \mathrm{min})$. Two catheters were inserted, one into the left 19 femoral artery for injection of compounds and nanoparticles, and for monitoring mean 20 arterial blood pressure (MABP; Pressure Monitor BP-1, World Precision 21 Instruments), and the other into the femoral vein for anesthesia infusion during 22 imaging. The animal was turned to the prone position, the scalp was removed, the 23 periosteum was removed with a $\mathrm{FeCl}_{3}$-soaked cotton bud, and the exposed skull was 24 glued (Loctite Adhesives) to a custom-made metal head plate. A craniotomy was 25 performed over the right somatosensory cortex $(3 \mathrm{~mm}$ lateral, $0.5 \mathrm{~mm}$ posterior to 26 bregma; $\emptyset=4 \mathrm{~mm} ; 4500 \mathrm{rpm}$ dental drill). The bone flap was carefully lifted, the dura 27 removed, and 1\% agarose (type III-A, Sigma-Aldrich) in artificial cerebrospinal fluid 28 (aCSF; in mM: $\mathrm{NaCl} 120, \mathrm{KCl} 2.8, \mathrm{Na}_{2} \mathrm{HPO}_{4} 1, \mathrm{MgCl}_{2} 0.876, \mathrm{NaHCO}_{3} 22, \mathrm{CaCl}_{2}$ 29 1.45, glucose 2.55, $\mathrm{pH}=7.4$ ) was applied on the brain surface. An imaging coverslip 30 ( 4×4 mm, 0.08-mm thick; Menzel-Gläser) was positioned onto the craniotomy, 31 leaving a $\sim 0.5$-mm gap for glass microelectrode insertion. The animal was transferred 32 to the imaging stage, and the anesthesia was changed to continuous infusion of $\alpha$ 33 chloralose $(50 \mathrm{mg} / \mathrm{kg} \mathrm{BW}$ per hour) via an intravenous catheter. 
1 Mice were allowed to rest for $25 \mathrm{~min}$ before the imaging procedures. Prior to 2 imaging, a $\sim 50 \mu \mathrm{L}$ blood sample was collected via the arterial catheter for blood gas

3 evaluation (ABL, Radiometer), and the respiration rate and volume were adjusted if

4 necessary. To ensure physiological conditions, we monitored end-tidal $\mathrm{CO}_{2}$ levels and

$5 \mathrm{MABP}$, and body temperature was maintained at $37^{\circ} \mathrm{C}$ using a rectal thermistor-

6 regulated heating pad.

\section{Animal preparation for awake/chronic awake imaging}

9 The surgery was performed as previously described, with minor modifications

10 (Holtmaat et al., 2009). Briefly, $4 \mathrm{~h}$ prior to the surgery, the animals were injected 11 with dexamethasone (4.8 mg/g BW; Dexavit, Vital Pharma Nordic). The anesthesia was induced with 5\% isoflurane (ScanVet) in $\mathrm{O}_{2}$-supplemented air (10\%). Eyes were lubricated with eye ointment (Viscotears, Novartis), and the animal's head was shaved and mounted onto a stereotactic frame. The body temperature was maintained during all steps of the surgery at $37^{\circ} \mathrm{C}$ using a rectal thermistor-regulated heating pad. Surgery was performed in an aseptic environment with heat-sterilized surgical tools.

17 The shaved skin was disinfected with chlorhexidine/alcohol (0.5\%/74\%; Kruuse).

18 Next, carprofen (5 mg/kg BW; Norodyl, Norbrook), buprenorphine (0.05 mg/kg BW;

19 Temgesic, Indivior), and lidocaine (100 $\mu \mathrm{L} 0.5 \%)$ were subcutaneously injected under the scalp. The anesthesia was reduced to $1.8 \%-2.0 \%$ isoflurane, the scalp was removed, and the bone surface was cleaned from the periosteum with an ultrasonic drill (Piezosurgery, Mectron). A craniotomy was performed over the right somatosensory cortex ( $2 \mathrm{~mm}$ lateral, $0.5 \mathrm{~mm}$ posterior to bregma; $\varnothing=3 \mathrm{~mm}$ ), the bone flap was carefully lifted, and the exposed brain temporarily covered with a hemostatic absorbable gelatin sponge (Spongostan $®$, Ferrosan, Denmark) pre-wetted with icecold aCSF. The cranial opening was filled with aCSF, then sealed with an autoclavesterilized round imaging coverslip $(\varnothing=4 \mathrm{~mm}, 0.17$-mm thick; Laser Micromachining LTD). The rim of the coverslip was secured with a thin layer of Vetbond (3M), and a lightweight stainless steel head plate (Neurotar) was positioned on the top of the skull in alignment with the cranial window. The skull was coated with adhesive resin cement (RelyX Ultimate, 3M) to secure the exposed bone, including skin incision rim, and to firmly attach the metal plate to the head. Next, the animals were transferred onto a pre-warmed heating pad to wake from anesthesia $(\sim 5 \mathrm{~min})$ in a cage

34 supplemented with pre-wetted food pellets for easy chow and hydration. 
1 Post-operation care consisted of subcutaneous injections of Temgesic $(3 \mathrm{~h})$ and

2 Rimadyl (24 and $48 \mathrm{~h}$ post-surgery, doses as before). Animal welfare was closely

3 monitored during the 7 days of post-surgical recovery and subsequent imaging

4 training procedures. All animals underwent recurrent 30-min/day training sessions

5 before the imaging to gradually habituate to the mobile cage system (Neurotar) with

6 sugar-supplemented water as a reward ( 14 days training). Given that no catheters

7 were mounted in chronically imaged animals, the nanoparticles were injected

8 retroorbitally 10 days post-surgery during brief ( 2 $\mathrm{min})$ isoflurane anesthesia (5\%).

9 This administration route was preferential to, e.g., tail vein injections, because it

10 provided better control over the injectant volume. The imaging sessions never

11 exceeded 45 min.

12

13 Nanoparticle preparation

14 1,2-Distearoyl-sn-glycero-3-phosphocholine (DSPC), ovine cholesterol, 1,215 distearoyl-sn-glycero-3-phosphoethanolamine-N-[methoxy(polyethylene glycol)16 2000] ammonium salt (DSPE-PEG2k), and 1,2-distearoyl-sn-glycero-317 phosphoethanolamine- $\mathrm{N}$-[maleimide(polyethylene glycol)-2000] ammonium salt 18 (DSPE-PEG2k-maleimide) were purchased from Avanti Polar Lipids (Alabaster, AL,

19 USA). The stealth nanoparticles were prepared to consist of DSPC/cholesterol/DSPE-

20 PEG2k (molar composition: 56.3:38.2:5.5), and the antibody-functionalized 21 nanoparticles to consist of DSPC/cholesterol/DSPE-PEG2k/DSPE-PEG2k-maleimide 22 (molar composition: 56.3:38.2:5:0.5). The fluorescent nanoparticles were 23 supplemented with $0.5 \mathrm{~mol} \%$ of 1,2-dipalmitoyl-sn-glycero-3-phosphoethanolamine 24 labeled with Atto488 (Atto488 DPPE) or Atto550 (Atto550 DPPE; Atto-Tec, Siegen, 25 Germany). To obtain lipid powder mixtures of the above compositions, the 26 constituent lipids were dissolved in tert-butanol (Sigma-Aldrich, St. Louis, MO, 27 USA)/Milli-Q water solution $(9: 1)$ and heated to $40-50^{\circ} \mathrm{C}$ to ensure complete 28 dissolution. The lipid solutions were then plunge-frozen in liquid $\mathrm{N}_{2}$ and lyophilized 29 overnight to remove the solvent (ScanVac CoolSafe lyophilizer, LaboGene, Allerød, 30 Denmark). 
1 To obtain fluorescently labeled nanoparticles, the lyophilized lipids were hydrated in

$270^{\circ} \mathrm{C}$ phosphate-buffered saline (PBS; $10 \mathrm{mM}$ phosphate, $137 \mathrm{mM}$ sodium chloride,

$32.7 \mathrm{mM} \mathrm{KCl}, \mathrm{pH} 7.4$; tablets from Sigma-Aldrich) to a $50 \mathrm{mM}$ lipid concentration.

4 The lipid suspensions were vortexed seven times in 5-min intervals, then subjected to

5 five freeze-thaw cycles by alternate placement in a liquid $\mathrm{N}_{2}$ and a $70^{\circ} \mathrm{C}$ water bath.

6 Next, the lipid suspensions were extruded 21 times through a 100-nm polycarbonate

7 filter (Whatman, GE Healthcare) at $70^{\circ} \mathrm{C}$ using a mini-extruder (Avanti Polar Lipids)

8 to form nanoparticles.

9

\section{Nanoparticle targeting}

11 We used a high-affinity $\left(\mathrm{K}_{\mathrm{D}}=6 \mathrm{nM}\right)$ monoclonal anti-TfR antibody clone RI7217 to 12 functionalize nanoparticles (Johnsen et al., 2018). The antibody was produced in13 house using the hybridoma technique at Laboratory for Neurobiology, Aalborg 14 University, Denmark. The antibody specificity was previously determined using 15 surface plasmon resonance (Johnsen et al., 2018). To functionalize the nanoparticles 16 with either RI7217 or a rat isotype IgG control (Thermo Fisher Scientific, Waltham, 17 MA, USA), we prepared solutions of $8 \mathrm{mg} / \mathrm{mL}$ antibody in borate buffer $(100 \mathrm{mM}$ 18 borate, $2 \mathrm{mM}$ EDTA, $\mathrm{pH}$ 8.0; all Sigma-Aldrich). The antibody concentrations were 19 determined from the absorbance at $280 \mathrm{~nm}$ (NanoDrop 2000c spectrophotometer, 20 NanoDrop Products, Thermo Fisher Scientific) using mass extinction coefficients of $211.3(\mathrm{mg} / \mathrm{mL})^{-1} \mathrm{~cm}^{-1}$ and $1.5(\mathrm{mg} / \mathrm{mL})^{-1} \mathrm{~cm}^{-1}$ for RI7217 and IgG isotype, respectively, 22 determined in a separate micro-BCA experiment. Traut's reagent (Thermo Fisher 23 Scientific) was added to a reagent-to-antibody molar ratio 10:1 in Protein LoBind 24 tubes (Eppendorf, Hamburg, Germany), and the solutions were incubated for $1 \mathrm{~h}$ at 25 room temperature under constant shaking at $500 \mathrm{rpm}$. Using Amicon Ultra-4 $30 \mathrm{kDa}$ 26 centrifugal filter units (Merck, Darmstadt, Germany), we transferred the thiolated 27 antibodies to PBS and determined their concentration using the NanoDrop 2000c as 28 described above. Next, we added $1.05 \mathrm{mg}$ of newly prepared thiolated antibodies to $700 \mu \mathrm{L}$ of newly prepared nanoparticles (lipid concentration $\sim 35-40 \mathrm{mM}$ ) in Protein

30 LoBind tubes and replaced the air phase in the tubes with $\mathrm{N}_{2}$. The samples were then 31 incubated for $24 \mathrm{~h}$ at room temperature under constant shaking at $500 \mathrm{rpm}$, allowing 32 the thiolated antibodies to couple to the maleimide groups the surface of the 33 nanoparticles. The antibody-functionalized nanoparticles were separated from 34 unbound antibodies using a Sepharose CL-4B (GE Healthcare, Little Chalfont, UK) 
1 size-exclusion chromatography column eluted with PBS (dimensions, $1.5 \times 20 \mathrm{~cm}$;

2 flow rate, $1 \mathrm{~mL} / \mathrm{min}$ ). The recovered nanoparticles were pooled in Amicon Ultra-4

$3100 \mathrm{kDa}$ centrifugal filter units (Merck) and concentrated by centrifuging at $2000 \times g$

4 until the lipid concentration was increased to $30-40 \mathrm{mM}$.

5

6 Nanoparticle cisplatin loading, targeting and detection.

7 To prepare cisplatin-loaded nanoparticles, cis-diammineplatinum(II) dichloride

8 (cisplatin; Sigma-Aldrich) was dissolved in PBS to a nominal concentration of 8.5

$9 \mathrm{mg} / \mathrm{mL}$ (Peleg-Shulman et al., 2001). The solutions were magnetically stirred for $1 \mathrm{~h}$

10 at $70^{\circ} \mathrm{C}$ and subsequently left at room temperature for $15 \mathrm{~min}$, allowing undissolved

11 cisplatin crystals to precipitate. The supernatants were transferred to new vials and 12 magnetically stirred while being heated to $70^{\circ} \mathrm{C}$. The solutions were then added to 13 lyophilized lipids, resulting in $50 \mathrm{mM}$ lipid suspensions that were magnetically stirred 14 for $1 \mathrm{~h}$ at $70^{\circ} \mathrm{C}$ and extruded as described above for the fluorescently labeled 15 nanoparticles. The samples were cooled to room temperature to allow any residual 16 cisplatin crystals to precipitate, and the supernatants were run on a Sepharose CL-4B 17 size-exclusion chromatography column eluted with PBS (dimensions $1.5 \times 20 \mathrm{~cm}$, flow 18 rate $1 \mathrm{~mL} / \mathrm{min}$ ) to remove free cisplatin. The recovered nanoparticles were 19 concentrated using Amicon Ultra-4 $100 \mathrm{kDa}$ centrifugal filter units by centrifuging at $202000 \times g$.

21 To prepare antibody-functionalized cisplatin-loaded nanoparticles, the antibodies 22 were thiolated as described above. Then, $0.5 \mathrm{mg}$ of newly prepared thiolated antibody 23 was added to $700 \mu \mathrm{L}$ of newly prepared cisplatin-loaded nanoparticles (lipid 24 concentration $\sim 13 \mathrm{mM}$ ) in a Protein LoBind tube. The nanoparticles were finally 25 incubated, recovered, and concentrated as described above for the fluorescently 26 labeled antibody-functionalized nanoparticles.

27 Brain uptake of cisplatin was measured using ICP-MS as recently described (Johnsen 28 et al., 2019a).

\section{$30 \quad$ Nanoparticle properties in vitro}

31 The phosphorus concentrations of the liposome stock solutions were determined using 32 inductively coupled plasma mass spectrometry (iCAP Q ICP-MS, Thermo Fisher 33 Scientific). We estimated phospholipid concentrations by subtracting the phosphorus 34 background of the PBS buffer and estimated total lipid concentrations by dividing the 
1 phospholipid concentrations by 0.618 , taking into account that cholesterol does not 2 contain phosphorus. For the cisplatin-loaded nanoparticles, we also used ICP-MS to 3 determine the platinum concentrations. The size of the nanoparticles (dissolved in 4 PBS) was measured using dynamic light scattering, and the zeta potential of the 5 nanoparticles in phosphate-glucose buffer (10 mM phosphate, $280 \mathrm{mM}$ glucose, $\mathrm{pH}$ 6 7.4; reagents from Sigma-Aldrich) was measured using mixed measurement mode 7 phase analysis light scattering (Zetasizer Nano ZS, Malvern Instruments, Malvern, 8 UK). The antibody conjugation level on the functionalized nanoparticle was 9 determined using the micro-BCA assay (reagents purchased from Thermo Fisher 10 Scientific), performed by incubating samples (including bovine serum albumin [BSA] 11 standard samples) for $1 \mathrm{~h}$ in a $60^{\circ} \mathrm{C}$ water bath and then transferring them to a 96-well 12 plate to measure their absorbance at $562 \mathrm{~nm}$ using a Spark multimode microplate 13 reader (Tecan, Männedorf, Switzerland). To account for the small contribution of 14 lipids in the micro-BCA assay (Kessler and Fanestil, 1986; Kristensen et al., 2019), 15 we also performed the micro-BCA on non-functionalized nanoparticles, which 16 allowed for the subtraction of the lipid contribution to determine the amount of 17 antibody conjugated to the nanoparticles. The hydrodynamic diameter $\left(\mathrm{D}_{\mathrm{h}}\right)$ of RI718 functionalized Atto 550-tagged (RI7-L-A550) and Atto 488-tagged (RI7-L-A488) 19 nanoparticles was in the range of $d_{h}=\sim 135-140 \mathrm{~nm}$ (Table S1) and comparable to 20 other TfR-targeted clinically relevant formulations (Johnsen and Moos, 2016; Lam et 21 al., 2018). Both RI7-L-A550 and RI7-L-A488 had a low polydispersity index ( $\leq 0.13)$, 22 indicating high size homogeneity. Assuming the nanoparticles contained on average $23 \sim 2.5 \times 10^{5}$ lipids, the conjugation level of $30 \mathrm{~g} / \mathrm{mol}_{\text {lipid }}$ corresponded to $\sim 50$ antibodies 24 per nanoparticle (Kristensen et al., 2019).

\section{Nanoparticle systemic interactions in vivo}

27 In contrast to other TfR ligands, RI7217 does not compete with endogenous

28 transferrin, and TfR vascular expression is highly specific to the brain (van Rooy et al., 2011). We observed no pathological changes in exhaled $\mathrm{CO}_{2}$ levels, mean arterial 30 blood pressure MABP, or brain activity after nanoparticle administration in acute 31 experiments (Figure S1). Furthermore, we detected no atypical behavior, weight loss, 32 or signs of neuroinflammation in animals monitored up to $48 \mathrm{~h}$ post-injection in 33 chronic imaging experiments. 
2 Other fluorescent probes

3 FITC-dextran (MW 10 kDa, 0.5\%, Sigma-Aldrich), TRITC-dextran (MW 65 kDa,

$41 \%$, Sigma-Aldrich), or bovine serum albumin Alexa Fluor 488-conjugate (BSA-

5 Alexa 488, 1\%, Invitrogen) was administered as a single bolus injection (50 $\mu \mathrm{L})$ via

6 a femoral arterial catheter. All were dissolved in sterile saline and administered

7 subsequently to nanoparticles. In addition to delineating a vessel lumen, lack of

8 extravascular leak of dyes indicated preserved BBB structural integrity after the

9 microsurgery.

\section{Imaging setup}

12 In vivo two-photon imaging was performed with a SP5 upright laser scanning 13 microscope (Leica Microsystems) coupled to MaiTai Ti:Sapphire laser (Spectra14 Physics). The images were collected using a $20 \times 1.0$ water-immersion objective. The 15 fluorescence signal was split by FITC/TRITC filter and collected by two separate 16 multi-alkali photomultipliers after $525-560 \mathrm{~nm}$ and $560-625 \mathrm{~nm}$ bandpass filter 17 (Leica Microsystems). The fluorophores were excited at $870 \mathrm{~nm}$ with the $14 \mathrm{mWatt}$ 18 output power at the sample. The images were collected using LAS AF v. 4.4 (Leica 19 Microsystems) in 16-bit color depth and exported to ImageJ for further analysis (v. $201.52 \mathrm{a} ; \mathrm{NIH}) .3 \mathrm{D}$ reconstructions were performed via volume rendering in Amira v. 6 21 (FEI Visualization Sciences Group).

\section{Surface density calculation}

24 To assess the spatio-temporal properties of nanoparticles association to the 25 endothelium, we monitored the association of nanoparticles for $2 \mathrm{~h}$ after injection 26 with respect to all cerebral vessel types using hyperstack (4-dimensional) imaging. 27 Data were recorded as a series of Z-stacks in bidirectional scanning mode with triple 28 frame averaging, from $387.5 \mu \mathrm{m} \times 387.5 \mu \mathrm{m}$ area $(2048 \times 2048$ pixel resolution) and $29144 \mu \mathrm{m}$ depth span (Z-step size $2.50 \mu \mathrm{m}$ ) with 7.5-min intervals between consecutive 30 Z-stacks. The cerebral microvessels were classified as pial arterioles or venules, 31 penetrating arterioles, post-capillary venules, ascending venules, or capillaries based 32 on their morphology and second harmonics generation (Grubb et al., 2020; Janiurek 33 et al., 2019). The nanoparticles were counted from all vessels in the field of view, 
1 with each individual vessel followed over time. The vessel surface area was

2 calculated from vessel diameter delineated by FITC-dx or TRITC-dx signal and the

3 length of the vessel measured in 3D. The association density was obtained from a

4 nanoparticle count per corresponding vessel surface area [nanoparticles $/ \mu \mathrm{m}^{2}$ ].

5

\section{Subcellular distribution mapping}

7 We imaged the surface of the vessels, i.e., a $\sim 5 \mu \mathrm{m}$ planar optical section aligned 8 with the vessel circumference. When measuring distances from the nucleus 9 geometric center to nanoparticles, we set the distance cut-off point to $11 \mu \mathrm{m}$ to

10 exclude the nanoparticles that belonged to neighboring endothelial cells and to avoid 11 distribution bias due to the non-concentric spindle-like geometry of endothelial cells. 12 In addition, we excluded nanoparticles located in line from the geometric center 13 towards the vessel wall, where the cut-off distance exceeded the vessel boundary. 14 We took this step to minimize the effect of the vessel curvature on the estimation of 15 the distance.

16

\section{MSD analysis}

18 To characterize nanoparticle movement dynamics, we used the mean square 19 displacement (MSD) analysis (Weimann et al., 2013). Time-lapse recordings were 20 collected in bidirectional scanning mode from $387.5 \mu \mathrm{m} \times 387.5 \mu \mathrm{m}$ area $(2048 \times 2048$ 21 pixel resolution) for $30 \mathrm{~min}$ at 30-s intervals (60 data timepoints). Each nanoparticle 22 trajectory was manually traced, treating the fluorescence radial symmetry center as a 23 nanoparticle location coordinate. The planar $(x, y)$ trajectories were projected to a 24 vector $(v)$ aligned with a vessel symmetry axis and with the direction of the blood 25 flow. For every trajectory, the displacements in $v$ coordinate in time $t$ were extracted 26 for each multiplier of the smallest resolved time interval $d$ (i.e., $t=d, t=2 d$, $27 t=3 d \ldots t=i^{*} d$ ), where $i=60$ timepoints and $d=30 \mathrm{~s}$. In simple model systems, the 28 significant deviation of $\operatorname{MSD}(\mathrm{t})$ from linearity with the increase of $t$ indicates a nonstochastic (directional) movement component (Weimann et al., 2013). We assessed

$30 \operatorname{MSDv}(t)$ linearity with the least squares linear regression fit weighted by the inverse 31 of data point variance (Michalet, 2010), followed by Wald-Wolfowitz runs test.

\section{Electrophysiological recordings}


1 Electrocortical brain activity $(\mathrm{ECoG})$ was recorded via a heat-pulled borosilicate glass

2 electrode containing an $\mathrm{Ag} / \mathrm{AgCl}$ filament and filled with aCSF (electrode tip $\emptyset, 2-3$

$3 \mu \mathrm{m}$; inner $\varnothing, 0.86 \mathrm{~mm}$; outer $\varnothing, 1.5 \mathrm{~mm}$; Sutter Instrument; resistance 1.5-2.0 M $\Omega$ ).

4 The electrode was inserted under the glass coverslip $\sim 50 \mu \mathrm{m}$ into the cerebral cortex,

5 and the reference electrode was positioned in the neck muscle. The total electrical

6 signal was filtered (3000 Hz low-pass filter), then amplified 10× (AP311 analog

7 amplifier; Warner Instruments), and the alternate current-ECoG component (i.e.,

8 spontaneous brain activity) was obtained after further $100 \times$ amplification and $0.5 \mathrm{~Hz}$

9 high-pass filter (NL 106 analog amplifier and NL 125/126 analog filter, NeuroLog).

10 Analog data were digitized (Power 1401, CED) at $20 \mathrm{kHz}$. For the exhaled $\mathrm{CO}_{2}$,

11 MABP (the raw readout) was collected. All data were recorded in Spike2 software (v.

12 7.02a; CED).

13

14 Statistical analysis

15 Following Pearson's normality test, either an unpaired two-tailed Student's t-test

16 with Welch's correction (normally distributed data) or Mann-Whitney test (non-

17 normally distributed data) was used. All statistical analyses were performed in Prism

18 v.8.2 (GraphPad). The sample size was selected based on our previous two-photon in

19 vivo experiments (Kucharz and Lauritzen, 2018). Data was plotted in Prism v.8.2. or

20 in OriginPro 2018 (OriginLab Corporation).

\section{Exclusion criteria}

23 Prior to injection of nanoparticles, all animals with abnormal blood pressure $(<50$

$24 \mathrm{mmHg}$ ), abnormal brain ECoG activity, or significant ( $>2 \mu \mathrm{m} / \mathrm{min}$ ) brain movement

25 in $x, y$, or $z$ coordinates were excluded from analysis ( 3 of 48 animals).

26

27

28

29

30

31

32

33

\section{REFERENCES}

Abbott, N.J., Patabendige, A.A., Dolman, D.E., Yusof, S.R., and Begley, D.J. (2010). Structure and function of the blood-brain barrier. Neurobiol Dis 37, 13-25.

Banks, W.A. (2016). From blood-brain barrier to blood-brain interface: new opportunities for CNS drug delivery. Nat Rev Drug Discov 15, 275-292. 
1 Barenholz, Y. (2012). Doxil(R)--the first FDA-approved nano-drug: lessons learned. J Control

2 Release 160, 117-134.

3 Ben-Zvi, A., Lacoste, B., Kur, E., Andreone, B.J., Mayshar, Y., Yan, H., and Gu, C. (2014). Mfsd2a is

4 critical for the formation and function of the blood-brain barrier. Nature 509, 507-511.

5 Cabezon, I., Manich, G., Martin-Venegas, R., Camins, A., Pelegri, C., and Vilaplana, J. (2015).

6 Trafficking of Gold Nanoparticles Coated with the 8D3 Anti-Transferrin Receptor Antibody at the

7 Mouse Blood-Brain Barrier. Mol Pharm 12, 4137-4145.

8 Chen, Y., and Liu, L. (2012). Modern methods for delivery of drugs across the blood-brain barrier.

9 Adv Drug Deliv Rev 64, 640-665.

10 Cheng, M.J., Kumar, R., Sridhar, S., Webster, T.J., and Ebong, E.E. (2016). Endothelial glycocalyx

11 conditions influence nanoparticle uptake for passive targeting. Int J Nanomedicine 11, 3305-

123315.

13 Choi, M., Ku, T., Chong, K., Yoon, J., and Choi, C. (2011). Minimally invasive molecular delivery into 14 the brain using optical modulation of vascular permeability. Proc Natl Acad Sci U S A 108, 9256-

159261.

16 Clark, A.J., and Davis, M.E. (2015). Increased brain uptake of targeted nanoparticles by adding an 17 acid-cleavable linkage between transferrin and the nanoparticle core. Proc Natl Acad Sci U S A $18 \quad 112,12486-12491$.

19 Claudio, L., Kress, Y., Factor, J., and Brosnan, C.F. (1990). Mechanisms of edema formation in 20 experimental autoimmune encephalomyelitis. The contribution of inflammatory cells. Am J 21 Pathol 137, 1033-1045.

22 Couch, J.A., Yu, Y.J., Zhang, Y., Tarrant, J.M., Fuji, R.N., Meilandt, W.J., Solanoy, H., Tong, R.K., Hoyte, 23 K., Luk, W., et al. (2013). Addressing safety liabilities of TfR bispecific antibodies that cross the 24 blood-brain barrier. Sci Transl Med 5, 183ra157, 181-112.

25 De Bock, M., Van Haver, V., Vandenbroucke, R.E., Decrock, E., Wang, N., and Leybaert, L. (2016).

26 Into rather unexplored terrain-transcellular transport across the blood-brain barrier. Glia 64,

$27 \quad 1097-1123$.

28 Engelhardt, B., Vajkoczy, P., and Weller, R.O. (2017). The movers and shapers in immune 29 privilege of the CNS. Nat Immunol 18, 123-131.

30 Faghih, M.M., and Sharp, M.K. (2018). Is bulk flow plausible in perivascular, paravascular and 31 paravenous channels? Fluids Barriers CNS 15, 17. 
1 Farias, G.G., Guardia, C.M., Britt, D.J., Guo, X., and Bonifacino, J.S. (2015). Sorting of Dendritic and

2 Axonal Vesicles at the Pre-axonal Exclusion Zone. Cell Rep 13, 1221-1232.

3 Foley, C.P., Nishimura, N., Neeves, K.B., Schaffer, C.B., and Olbricht, W.L. (2012). Real-time imaging

4 of perivascular transport of nanoparticles during convection-enhanced delivery in the rat cortex.

5 Ann Biomed Eng 40, 292-303.

6 Friden, P.M., Walus, L.R., Musso, G.F., Taylor, M.A., Malfroy, B., and Starzyk, R.M. (1991). Anti-

7 transferrin receptor antibody and antibody-drug conjugates cross the blood-brain barrier. Proc

$8 \quad$ Natl Acad Sci U S A 88, 4771-4775.

9 Ge, S., Song, L., and Pachter, J.S. (2005). Where is the blood-brain barrier ... really? J Neurosci Res $1079,421-427$.

11 Grant, B.D., and Donaldson, J.G. (2009). Pathways and mechanisms of endocytic recycling. Nat

12 Rev Mol Cell Biol 10, 597-608.

13 Gromnicova, R., Kaya, M., Romero, I.A., Williams, P., Satchell, S., Sharrack, B., and Male, D. (2016).

14 Transport of Gold Nanoparticles by Vascular Endothelium from Different Human Tissues. PLoS

15 One 11, e0161610.

16 Grubb, S., Cai, C., Hald, B.O., Khennouf, L., Murmu, R.P., Jensen, A.G.K., Fordsmann, J., Zambach, S., 17 and Lauritzen, M. (2020). Precapillary sphincters maintain perfusion in the cerebral cortex. Nat 18 Commun 11, 395.

19 Holtmaat, A., Bonhoeffer, T., Chow, D.K., Chuckowree, J., De Paola, V., Hofer, S.B., Hubener, M., 20 Keck, T., Knott, G., Lee, W.C., et al. (2009). Long-term, high-resolution imaging in the mouse 21 neocortex through a chronic cranial window. Nat Protoc 4, 1128-1144.

22 Janiurek, M., Soylu-Kucharz, R., Christoffersen, C., Kucharz, K., and Lauritzen, M. (2019).

23 Apolipoprotein M-bound sphingosine-1-phosphate regulates blood-brain barrier paracellular

24 permeability and transcytosis. Elife 8.

25 Jefferies, W.A., Brandon, M.R., Hunt, S.V., Williams, A.F., Gatter, K.C., and Mason, D.Y. (1984).

26 Transferrin receptor on endothelium of brain capillaries. Nature $312,162-163$.

27 Johnsen, K.B., Bak, M., Kempen, P.J., Melander, F., Burkhart, A., Thomsen, M.S., Nielsen, M.S., Moos,

28 T., and Andresen, T.L. (2018). Antibody affinity and valency impact brain uptake of transferrin

29 receptor-targeted gold nanoparticles. Theranostics $8,3416-3436$.

30 Johnsen, K.B., Bak, M., Melander, F., Thomsen, M.S., Burkhart, A., Kempen, P.J., Andresen, T.L., and

31 Moos, T. (2019a). Modulating the antibody density changes the uptake and transport at the 
1 blood-brain barrier of both transferrin receptor-targeted gold nanoparticles and liposomal cargo.

2 J Control Release 295, 237-249.

3 Johnsen, K.B., Burkhart, A., Thomsen, L.B., Andresen, T.L., and Moos, T. (2019b). Targeting the

4 transferrin receptor for brain drug delivery. Prog Neurobiol 181, 101665.

5 Johnsen, K.B., and Moos, T. (2016). Revisiting nanoparticle technology for blood-brain barrier

6 transport: Unfolding at the endothelial gate improves the fate of transferrin receptor-targeted

7 liposomes. J Control Release 222, 32-46.

8 Kessler, R.J., and Fanestil, D.D. (1986). Interference by lipids in the determination of protein using

9 bicinchoninic acid. Anal Biochem 159, 138-142.

10 Knowland, D., Arac, A., Sekiguchi, K.J., Hsu, M., Lutz, S.E., Perrino, J., Steinberg, G. K., Barres, B.A., 11 Nimmerjahn, A., and Agalliu, D. (2014). Stepwise recruitment of transcellular and paracellular

12 pathways underlies blood-brain barrier breakdown in stroke. Neuron 82, 603-617.

13 Kristensen, K., Engel, T.B., Stensballe, A., Simonsen, J.B., and Andresen, T.L. (2019). The hard

14 protein corona of stealth liposomes is sparse. J Control Release 307, 1-15.

15 Kucharz, K., and Lauritzen, M. (2018). CaMKII-dependent endoplasmic reticulum fission by

16 whisker stimulation and during cortical spreading depolarization. Brain 141, 1049-1062.

17 Kutuzov, N., Flyvbjerg, H., and Lauritzen, M. (2018). Contributions of the glycocalyx, endothelium, 18 and extravascular compartment to the blood-brain barrier. Proc Natl Acad Sci U S A 115, E9429-

$19 \quad$ E9438.

20 Lajoie, J.M., and Shusta, E.V. (2015). Targeting receptor-mediated transport for delivery of

21 biologics across the blood-brain barrier. Annu Rev Pharmacol Toxicol 55, 613-631.

22 Lam, F.C., Morton, S.W., Wyckoff, J., Vu Han, T.L., Hwang, M.K., Maffa, A., Balkanska-Sinclair, E.,

23 Yaffe, M.B., Floyd, S.R., and Hammond, P.T. (2018). Enhanced efficacy of combined temozolomide

24 and bromodomain inhibitor therapy for gliomas using targeted nanoparticles. Nat Commun 9 ,

251991.

26 Lee, H.J., Engelhardt, B., Lesley, J., Bickel, U., and Pardridge, W.M. (2000). Targeting rat anti-mouse

27 transferrin receptor monoclonal antibodies through blood-brain barrier in mouse. J Pharmacol

28 Exp Ther 292, 1048-1052.

29 Lindqvist, A., Friden, M., and Hammarlund-Udenaes, M. (2016). Pharmacokinetic considerations

30 of nanodelivery to the brain: Using modeling and simulations to predict the outcome of liposomal

31 formulations. Eur J Pharm Sci 92, 173-182. 
1 Mestre, H., Tithof, J., Du, T., Song, W., Peng, W., Sweeney, A.M., Olveda, G., Thomas, J.H.,

2 Nedergaard, M., and Kelley, D.H. (2018). Flow of cerebrospinal fluid is driven by arterial

3 pulsations and is reduced in hypertension. Nat Commun 9, 4878.

4 Michalet, X. (2010). Mean square displacement analysis of single-particle trajectories with

5 localization error: Brownian motion in an isotropic medium. Phys Rev E Stat Nonlin Soft Matter

6 Phys $82,041914$.

7 Mollgard, K., Dziegielewska, K.M., Holst, C.B., Habgood, M.D., and Saunders, N.R. (2017). Brain

8 barriers and functional interfaces with sequential appearance of ABC efflux transporters during

9 human development. Sci Rep 7,11603.

10 Moos, T., and Morgan, E.H. (2001). Restricted transport of anti-transferrin receptor antibody

11 (OX26) through the blood-brain barrier in the rat. J Neurochem 79, 119-129.

12 Motoike, T., Loughna, S., Perens, E., Roman, B.L., Liao, W., Chau, T.C., Richardson, C.D., Kawate, T.,

13 Kuno, J., Weinstein, B.M., et al. (2000). Universal GFP reporter for the study of vascular

14 development. Genesis 28, 75-81.

15 Muldoon, L.L., Pagel, M.A., Kroll, R.A., Roman-Goldstein, S., Jones, R.S., and Neuwelt, E.A. (1999). A

16 physiological barrier distal to the anatomic blood-brain barrier in a model of transvascular

17 delivery. AJNR Am J Neuroradiol 20, 217-222.

18 Nance, E.A., Woodworth, G.F., Sailor, K.A., Shih, T.Y., Xu, Q., Swaminathan, G., Xiang, D., Eberhart,

19 C., and Hanes, J. (2012). A dense poly(ethylene glycol) coating improves penetration of large

20 polymeric nanoparticles within brain tissue. Sci Transl Med 4, 149 ra119.

21 Niewoehner, J., Bohrmann, B., Collin, L., Urich, E., Sade, H., Maier, P., Rueger, P., Stracke, J.O., Lau,

22 W., Tissot, A.C., et al. (2014). Increased brain penetration and potency of a therapeutic antibody

23 using a monovalent molecular shuttle. Neuron 81, 49-60.

24 Pardridge, W.M. (2002). Drug and gene delivery to the brain: the vascular route. Neuron 36, 555-

25558.

26 Pardridge, W.M. (2012). Drug transport across the blood-brain barrier. J Cereb Blood Flow Metab $27 \quad 32,1959-1972$.

28 Pardridge, W.M. (2020). Blood-Brain Barrier and Delivery of Protein and Gene Therapeutics to 29 Brain. Front Aging Neurosci 11, 373.

30 Peleg-Shulman, T., Gibson, D., Cohen, R., Abra, R., and Barenholz, Y. (2001). Characterization of 31 sterically stabilized cisplatin liposomes by nuclear magnetic resonance. Biochim Biophys Acta $32 \quad 1510,278-291$. 
1 Pulgar, V.M. (2018). Transcytosis to Cross the Blood Brain Barrier, New Advancements and

2 Challenges. Front Neurosci 12, 1019.

3 Santisakultarm, T.P., Cornelius, N.R., Nishimura, N., Schafer, A.I., Silver, R.T., Doerschuk, P.C.,

4 Olbricht, W.L., and Schaffer, C.B. (2012). In vivo two-photon excited fluorescence microscopy

5 reveals cardiac- and respiration-dependent pulsatile blood flow in cortical blood vessels in mice.

6 Am J Physiol Heart Circ Physiol 302, H1367-1377.

7 Soldati, T., and Schliwa, M. (2006). Powering membrane traffic in endocytosis and recycling. Nat

8 Rev Mol Cell Biol 7, 897-908.

9 Tarasoff-Conway, J.M., Carare, R.O., Osorio, R.S., Glodzik, L., Butler, T., Fieremans, E., Axel, L.,

10 Rusinek, H., Nicholson, C., Zlokovic, B.V., et al. (2015). Clearance systems in the brain-implications

11 for Alzheimer disease. Nat Rev Neurol 11, 457-470.

12 Thomsen, M.S., Routhe, L.J., and Moos, T. (2017). The vascular basement membrane in the healthy 13 and pathological brain. J Cereb Blood Flow Metab 37, 3300-3317.

14 Thorne, R.G., and Nicholson, C. (2006). In vivo diffusion analysis with quantum dots and dextrans 15 predicts the width of brain extracellular space. Proc Natl Acad Sci U S A 103, 5567-5572.

16 Tietz, S., and Engelhardt, B. (2015). Brain barriers: Crosstalk between complex tight junctions

17 and adherens junctions. J Cell Biol 209, 493-506.

18 Tonnesen, J., Inavalli, V., and Nagerl, U.V. (2018). Super-Resolution Imaging of the Extracellular

19 Space in Living Brain Tissue. Cell 172, 1108-1121 e1115.

20 Tsai, P.S., Kaufhold, J.P., Blinder, P., Friedman, B., Drew, P.J., Karten, H.J., Lyden, P.D., and Kleinfeld,

21 D. (2009). Correlations of neuronal and microvascular densities in murine cortex revealed by

22 direct counting and colocalization of nuclei and vessels. J Neurosci 29, 14553-14570.

23 van Gelder, W., Huijskes-Heins, M.I., van Dijk, J.P., Cleton-Soeteman, M.I., and van Eijk, H.G.

24 (1995). Quantification of different transferrin receptor pools in primary cultures of porcine

25 blood-brain barrier endothelial cells. J Neurochem 64, 2708-2715.

26 van Rooy, I., Mastrobattista, E., Storm, G., Hennink, W.E., and Schiffelers, R.M. (2011). Comparison

27 of five different targeting ligands to enhance accumulation of liposomes into the brain. J Control

28 Release 150, 30-36.

29 Wardlaw, J.M., Benveniste, H., Nedergaard, M., Zlokovic, B.V., Mestre, H., Lee, H., Doubal, F.N.,

30 Brown, R., Ramirez, J., MacIn tosh, B.J., et al. (2020). Perivascular spaces in the brain: anatomy,

31 physiology and pathology. Nat Rev Neurol 16, 137-153. 
1 Weimann, L., Ganzinger, K.A., McColl, J., Irvine, K.L., Davis, S.J., Gay, N.J., Bryant, C.E., and

2 Klenerman, D. (2013). A quantitative comparison of single-dye tracking analysis tools using

3 Monte Carlo simulations. PLoS One 8, e64287.

4 Weller, R.O., Sharp, M.M., Christodoulides, M., Carare, R.O., and Mollgard, K. (2018). The meninges

5 as barriers and facilitators for the movement of fluid, cells and pathogens related to the rodent

6 and human CNS. Acta Neuropathol 135, 363-385.

7 Wiley, D.T., Webster, P., Gale, A., and Davis, M.E. (2013). Transcytosis and brain uptake of

8 transferrin-containing nanoparticles by tuning avidity to transferrin receptor. Proc Natl Acad Sci

9 U S A 110, 8662-8667.

10 Yu, Y.J., Zhang, Y., Kenrick, M., Hoyte, K., Luk, W., Lu, Y., Atwal, J., Elliott, J.M., Prabhu, S., Watts, R.J., 11 et al. (2011). Boosting brain uptake of a therapeutic antibody by reducing its affinity for a 12 transcytosis target. Sci Transl Med 3, 84ra44.

13 Zhang, E.T., Inman, C.B., and Weller, R.O. (1990). Interrelationships of the pia mater and the 14 perivascular (Virchow-Robin) spaces in the human cerebrum. J Anat 170, 111-123.

15

16

17

18

\section{Figure 1. Two-photon imaging of liposome nanoparticles in vivo.}
A) Mouse after the preparative surgery for acute imaging.
B) Features of an acute cranial window.
C) Principal components of targeted nanoparticles used in the study.

D) RI7217-functionalized nanoparticles drive accumulation of cisplatin payload in the brain, in contrast to nanoparticles functionalized with isotype IgGs or devoid of antibody functionalization (stealth liposomes). \% ID/g is percentage of injected dose of encapsulated cisplatin per gram tissue; $n=4$ mice.

E) 3D reconstruction of the brain microvasculature delineated by circulating nanoparticles.

F) Time-lapse images of blood-circulating nanoparticles following injection into the bloodstream. Long panels are kymographs of fluorescence at demarked lines. See also Movie S1.

G) Upper panel: nanoparticle fluorescence signal over time. Lower panel: fluorescence signal from RI7-L-A550 and RI7-L-A488 nanoparticles after $2 \mathrm{~h}$ of continuous imaging matches the stability of FITC-dx and outperforms 
TRITC-dx, commonly used in imaging studies (ANOVA). $(n)=$ number of mice
H) Laser-extravasated nanoparticles into brain parenchyma after $3 \mathrm{~h}$ after injection into the circulation. Nanoparticles retained their discrete and homogeneous appearance.

I) Laser-extravasated RI7-L-A488 and RI7-LA550 into the brain parenchyma 3 $\mathrm{h}$ after injection into the circulatiion. No liposome fusion or aggregation occured when present in circulation or in the brain.

J) Average of fluorescence profile plots of nanoparticles laser-extravasated to the brain $3 \mathrm{~h}$ after injection into the circulation. The average standard deviation $(\sigma)$ of signal distribution did not differ between distinctively labeled nanoparticles. $(n)=$ number of nanoparticles.

$* \mathrm{p}<0.05 ; * * * \mathrm{p}<0.001, n s=$ non-significant. Data are means \pm SEM.

Figure 2. Robust association of targeted nanoparticles to capillaries and postcapillary venules.

A-E) In vivo images of RI7-L-A550 nanoparticles (red) in vivo $3 \mathrm{~h}$ after injection to the circulation. Co-injected circulating FITC-dx delineates vessel lumen (green). Liposomes associate with vessel walls at pial, ascending venules, post-capillary venules, and capillaries.

F) No association of liposomes to an arterial branch of the brain microvasculature.

G) Co-injection of both RI7-L-A550 and RI7-L-A488 revealed discrete punctae of both nanoparticles at the vessel walls.

H) Time-lapse images of laser-extravasated nanoparticles in brain parenchyma. The proximity of nanoparticles causes overlapping fluorescence signal.

$p V=$ pial venule; $a s c V=$ ascending venule; $p c V=$ post-capillary venule; $c a p=$ capillaries; pen $A=$ penetrating arteriole; $p A=$ pial arteriole.

Figure 3. Nanoparticle association density and kinetics are determined by vessel type.

A) The difference between RI7-L-A550 (red) and FITC-dx (green, vessel lumen) fluorescence signal used to show the fraction of non-circulating nanoparticles that contour vessel boundaries (RI7-L-A550nc, cyan). 
B) $3 \mathrm{D}$ reconstruction of the nanoparticles associatiod to endothelium $2 \mathrm{~h}$ postinjection. The recuruited (non-circulating) nanoparticles (RI7-L-A550nc, cyan) are superimposed on the signal from liposomes circulating in the bloodstream (gray).

C) Upper panel: brain microvasculature following co-injection of RI7-L-A550 nanoparticles (red) and FITC-dx (vessel lumen, green) into circulation. Squares indicate areas magnified in lower panels. Lower panels: association of nanoparticles over time. See also Movie S3.

D) Nanoparticle distribution $2 \mathrm{~h}$ post-injection. Each data point is a single vessel. Blue areas indicate vessel segments. Dashed line demarks lognormal distribution trendline. Inset illustrates vessel classification.

E) Nanoparticles associated most rapidly with capillaries and most slowly with large venules. Surface density=\# of nanoparticles per $\mu \mathrm{m}^{2}$ vessel wall area.

$p V=$ pial venule; $a s c V=$ ascending venule; $p c V=$ post-capillary venule; $c a p=$ capillaries; $p c A=$ pre-capillary arteriole; penA=penetrating arteriole; $p A=$ pial arteriole. Data are means \pm SEM.

Figure 4. Prevalence of internalized nanoparticles in the brain vasculature.

A) Brain endothelium in vivo imaged in Tie2-GFP mice.

B) Detailed morphology of endothelial cells in vivo with noticeable cell boundaries and bright nuclei.

C) Simultaneous imaging of the brain endothelium and nanoparticles $2 \mathrm{~h}$ after RI7-L-A550 injection into circulation.

D) Pial venule with a clear presence of nanoparticles. Boxes 1-4 represent magnified areas on the right panels. \#5 is the magnified area presented in E).

E) Upper panel: nanoparticles (red) at two distinct locations (I., II.) in relation to the endothelium (Tie2-GFP, green). Dashed lines indicate the axes of fluorescence profiles in the lower panel. Lower panel: fluorescence profiles of a nanoparticles with low (I.) and high signal overlap (II). $\Delta \mathrm{p}$ demarks signal peaks separation.

F) Examples of internalized $(|\Delta \mathrm{p}|<\sigma)$, unresolved $(\sigma<|\Delta \mathrm{p}|<2 \sigma)$, and adhering $(|\Delta \mathrm{p}|>2 \sigma)$ nanoparticles. $\sigma=0.29 \mu \mathrm{m}$. Scale bars are provided in both $\sigma$ and $\mu \mathrm{m}$. Numbers at arrowheads show $\Delta \mathrm{p}$ value in $\sigma$ units. 
G) Histogram of percentage distribution of internalized (int.), unresolved (unr.), and adhering ( $a d h$.) liposomes. Inset illustrates vessel type division. Values over horizontal lines are the sum of underneath bins. $n=$ number of liposomes, values in brackets are the number of mice. Histogram $\operatorname{bin}=\sigma=0.29 \mu \mathrm{m}$.

Image insets denote the position of imaging plane relative to a vessel perimeter. $m V=$ main pial venule; $p V=$ pial venule; asc $V=$ ascending venule; $p c V=$ post-capillary venule; $c a p=$ capillaries; $p e n A=$ penetrating arteriole; $p A=$ pial arteriole.

\section{Figure 5. Nanoparticles exhibit directional movement in all vessel types, but} distinct subcellular distribution.

A) Time-lapse images of nanoparticle movement in large venules. Left panel: vessel surface scan. Right panel: vessel cross-section scan. Arrowheads indicate the movement of a nanoparticle. See also Movie S4

B) Tracking of nanoparticles. Left panel: circles outline traced nanoparticles. Middle panel: movement traces during $30 \mathrm{~min}$ of continuous time-lapse imaging. Right panel: isolated movement traces (black) with contours delineating the microvessel (gray). See also Movie S5

C) Movement traces in $x, y$ (Cartesian) coordinate system with respect to a vessel type. $(0,0)$ corresponds to the initial position of a nanoparticle.

D) Translation of the movement in $x, y$ coordinates into the movement relative to the blood flow direction $v . v>0$ indicates the movement along the blood flow, and $v<0$ is against the blood flow direction.

E-F) Vessel type does not influence the total distance traveled and the displacement (end-position) of a nanoparticle. Time span=30 min. $n=$ number of nanoparticles, values in brackets are the number of mice.

G) Blood flow direction does not affect the direction of movement of nanoparticles in large (pial venules) and small microvessels (post-capillary venules and capillaries).

H) MSD analysis showing significant deviation of $\operatorname{MSD}(\mathrm{t})$ from linearity (WaldWolfowitz runs test). Solid line shows least squares linear regression fit.

I) Nanoparticles preferentially distributed to perinuclear areas of venules.

J) No preferential distribution in capillaries. 
K) Calculation of nanoparticles location (bold solid lines) in relation to nucleus perimeter (solid edges). Distance lines meet at the geometric center of a nucleus (dashed lines).

L) Density map of nanoparticle distribution in relation to the geometric center of the nucleus. Kernel=2 $\sigma$. The heat-map represents the probability of liposome presence at a given coordinate.

M) Nanoparticle distribution in relation to nucleus boundary (bin=0.5 $\mu \mathrm{m}$ ). Numbers over horizontal lines are the sum of underneath bins. Empty bars are liposomes overlapping with nucleus signal (non-classified). $n=$ number of liposomes; number in brackets indicates number of animals.

N) Nanoparticles do not distribute to the endothelial cells perimeter and contact sites. Dashed lines indicate cell boundaries.

Insets in images denote the position of imaging plane relative to a vessel perimeter. $p V=$ pial venule; $p c V=$ post-capillary venule; $c a p=$ capillary; $n u c=$ nucleus; $M S D v=$ mean squared displacement in $v$ coordinate. E-G) Data are medians with interquartile ranges (IQR). H) Data are means \pm SEM.

\section{Figure 6. Post-capillary venules is the locus for TfR-mediated transcytosis.}

A) Internalized nanoparticles located at the basolateral side of endothelium in a capillary and a pial venule.

B) Lateral nanoparticle movement in capillaries.

C) Movement of a nanoparticle perpendicular to the vessel lumen.

D) Nanoparticle crossing the endothelium into the perivascular space in venules. The images show a nanoparticle accelerating in the perivascular space. See also Movie S7.

E) Schematic drawing of a mouse head and craniotomy for chronic two-photon imaging.

F) Microscope stage with movement-unrestricted awake animal. The objective is stationary, and the air-pressurized pad reacts reciprocally to the mouse movement.

G) Time course of nanoparticle penetration to brain parenchyma. Images are Zstack maximum intensity projection. Upper rows: Nanoparticles enter the brain at post-capillary venular segments; Lower row: No nanoparticle 
transcytosis to the brain at capillaries. null=fluorescence signal prior to nanoparticles injection.

H) High-resolution images of nanoparticles upon transcytosis in pial, ascending and precapillary venules.

$p A=$ pial artery; $p V=$ pial venule; $p c V=$ post-capillary venule; $c a p=$ capillary.

Figure 7. Results summary.

\section{SUPPLEMENTAL FIGURES LEGENDS}

\section{Figure S1. No adverse systemic effects on physiology.}

A) Blood-circulating leukocytes with sequestered nanoparticles (arrowheads). See also Movie S2.

B) Leukocytes with sequestered nanoparticles preserve their rolling and endothelium adherence properties.

C) The uptake of nanoparticles is unlikely driven by the RI7217 moiety, as it is also present e.g. for fluorescently labeled albumin (BSA-Alexa488).

D) No significant effect of nanoparticles on brain activity (electrocorticogram, $E C o G)$, exhaled $\mathrm{CO}_{2}$ levels $\left(\mathrm{exCO}_{2}\right)$, and mean arterial blood pressure $(M A B P)$, all measured simultaneously with imaging.

Figure S2. No nanoparticle association without TfR-targeting moiety.

$$
\text { A-B) No association of stealth Atto 550-tagged nanoparticles (Sth-L-A550) to }
$$
pial vessels and capillaries.

C-D) No association of isotype IgG Atto 550-tagged nanoparticles (IgG-L-A550) to pial vessels and capillaries.

Figure S3. RI7-L-A488 follows RI7-L-A550 spatio-temporal distribution.

A-D) High resolution in vivo images of RI7-L-A488 nanoparticles (green) $2 \mathrm{~h}$ post-injection. Co-injected circulating TRITC-dx delineates vessel lumen (red). Liposomes are readily present at pial, ascending venules, post-capillary venules, and capillaries in vivo. 
E) No association of isotype IgG A488-tagged nanoparticles with pial vessels and capillaries.

F-G) Association of RI7-L-A488 over time in vivo. Nanoparticles associated most rapidly to endothelium of capillaries and most slowly to large venules. Surface density=\# of nanoparticles per $\mu \mathrm{m}^{2}$ vessel wall area. Inset illustrates vessel type classification.

$m V=$ main pial venule; $p V=$ pial venule; $a s c V=$ ascending venule; $p c V=$ post-capillary venule; $c a p=$ capillaries; $p A=$ pial arteriole.

\section{Figure S4. Two-photon imaging of brain microvasculature endothelium in vivo.}

A) Projected Z-stacks from Tie2-GFP mouse cortex showing brain microvasculature endothelium in vivo. The high-resolution scan details the subcellular morphology of arterioles and venules.

$p V=$ pial venule; $a s c V=$ ascending venule; $p c V=$ post-capillary venule; $c a p=$ capillaries; pen $A=$ penetrating arteriole; $\quad p A=$ pial arteriole; $n u c=$ nucleus; $\quad c s=$ cell boundaries/endothelium contact sites.

\section{SUPPLEMENTAL MOVIES LEGENDS:}

Movie S1. Blood-circulating nanoparticles show high fluorescence stability over time. Time is relative to the time of nanoparticle injection.

Movie S2. A small fraction of nanoparticles is sequestered by circulating leukocytes.

Movie S3. Nanoparticle association to the brain microvasculature over time. Concurrent imaging of RI7-L-A550 nanoparticles (red) with FITC-dextran (FITC-dx, green). Time is relative to the time of nanoparticle injection.

Movie S4. Time-lapse recording of nanoparticle (RI7-L-A550) movement in the brain endothelial cells. The endothelium is visible in green (Tie2-GFP). Left panels: pial venule; right panel: post-capillary venule. 
1 Movie S5. Time-lapse recording of nanoparticle (RI7-L-A550) motility at the 2 capillary segment with examples of nanoparticle tracing. Circles denote individual 3 nanoparticles.

4

$5 \quad$ Movie S6. Nanoparticles exhibit movement, even at stalled capillaries.

6

7 Movie S7. Right panel: nanoparticle (RI7-L-A550, red) transcytosis into the 8 perivascular space at the level of post-capillary venules. Left panel: no nanoparticle 9 transcytosis in capillaries. The vessel lumen is delineated by circulating FITC-dextran 10 (FITC-dx, green).

11

12 Movie S8. Leukocyte entry into the brain with previously sequestered nanoparticles 13 (RI7-L-A550, red) in the blood circulation. Vessel lumen is delineated by circulating 14 FITC-dextran (FITC-dx, green).

15

16 Movie S9. No significant nanoparticle movement following laser-extravasation from 17 the blood to the brain at capillaries. In contrast, rapid progression in the brain of 18 nanoparticles extravasated from venules. Lines denote vessel lumen boundaries. 


\section{$\mathbf{A}$}

RI7-L-A550 + FITC-dx
B

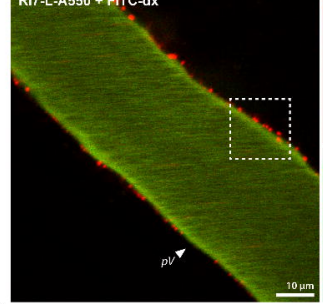

D

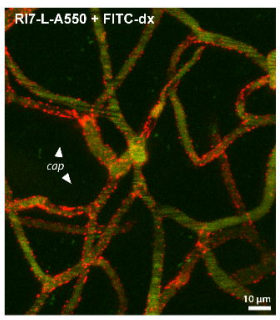

F

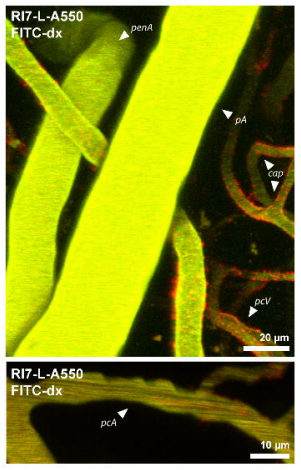

G

H RI7-L-A550
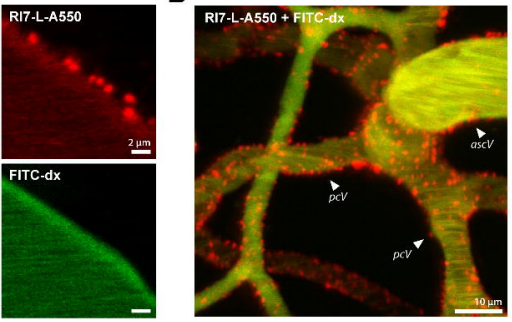

E

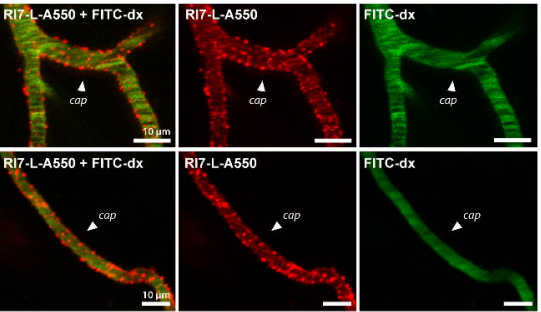

RI7-L-A550 + FITC-dx

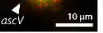

RI7-L-A550

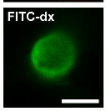

R17-L-A550 + FITC-dx

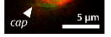

RI7-L-A550

RIT-A650
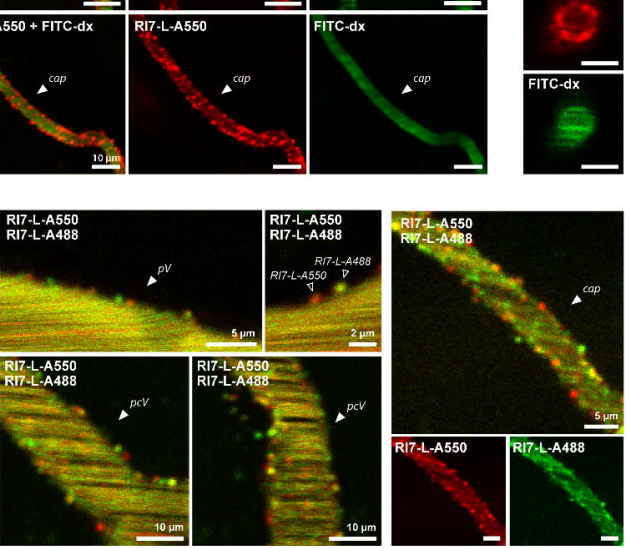

RI7-L-A550

Ri7-L-A488

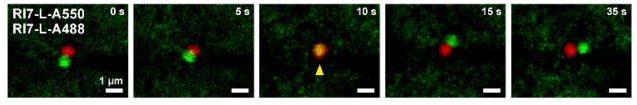




\section{A}
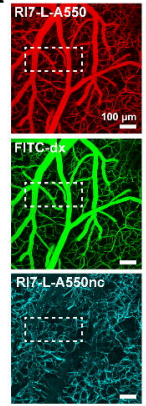

C
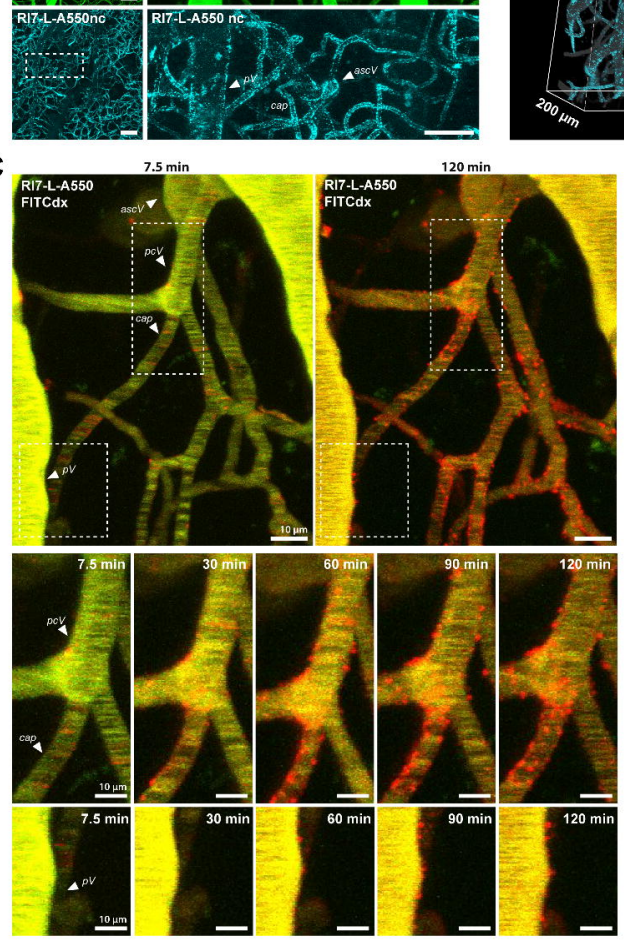

$20 \mu \mathrm{m}$
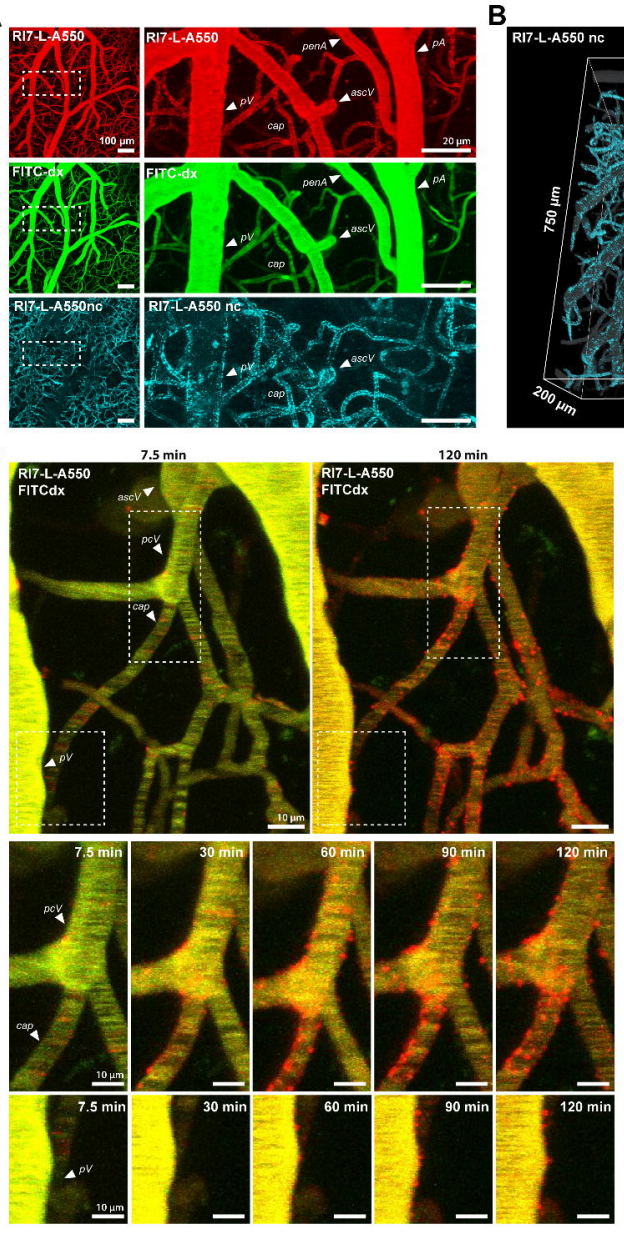

$750 \mu \mathrm{m}$

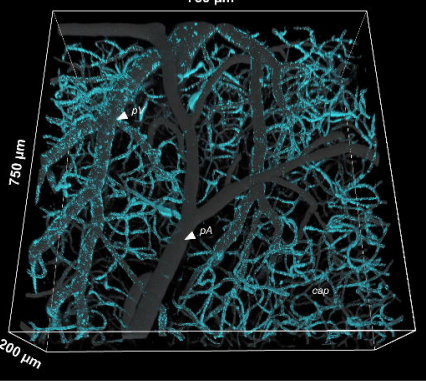

D
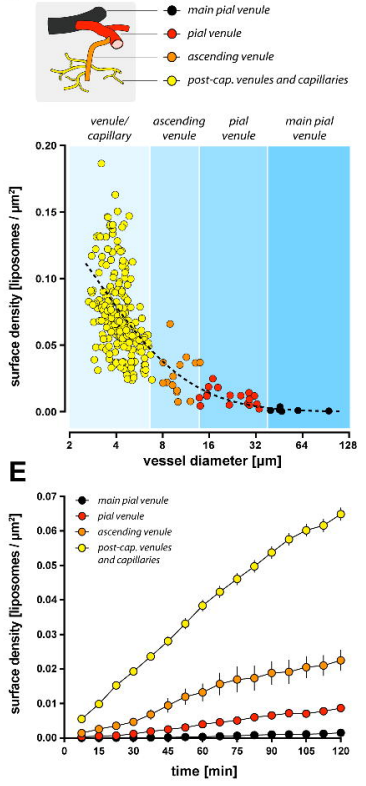


\section{A}

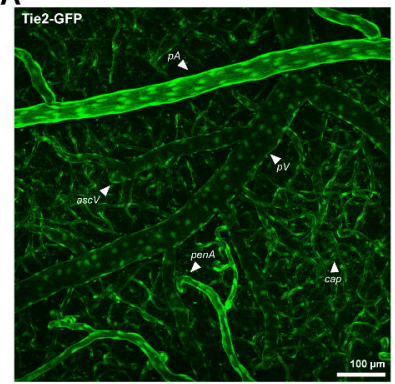

B
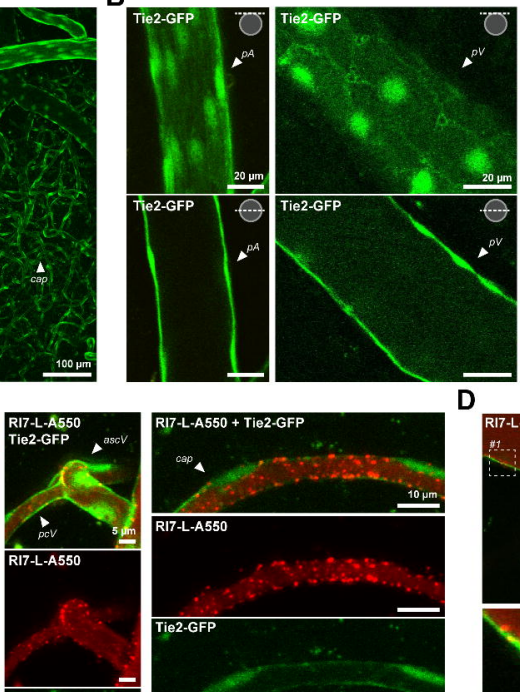

Tie2-GFP

E

F

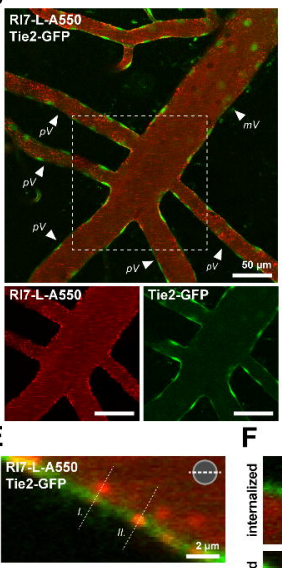

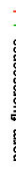

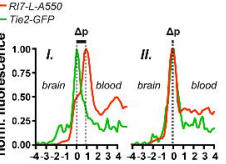

RI7 $7-L-A 550$ distance $[\mu \mathrm{m}]$

D

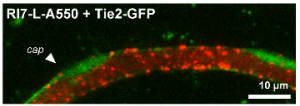

R17-L-A550

$10 \mu \mathrm{m}$

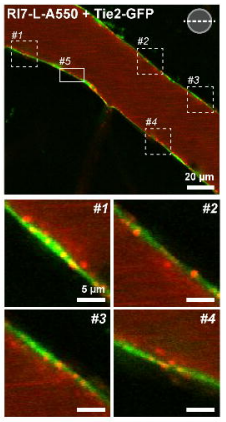

G
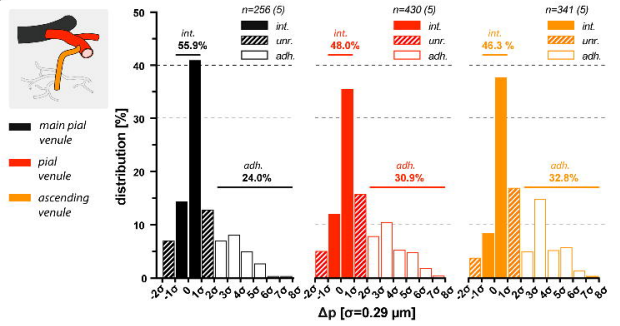


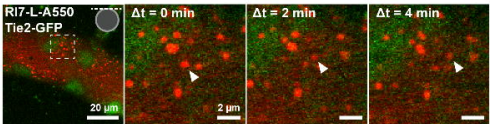

B
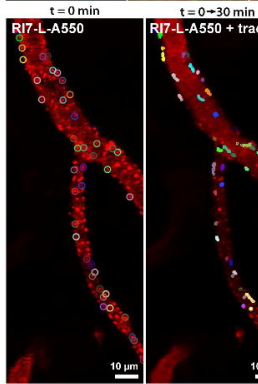

$\mathrm{t}=0 \rightarrow 30 \mathrm{~min}$ Rv7-L-A550 + traces isolated traces

C
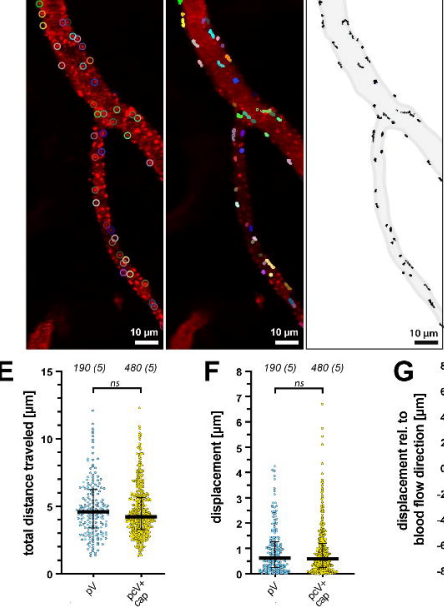

RI7-L-A550 THe2.GFP

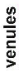
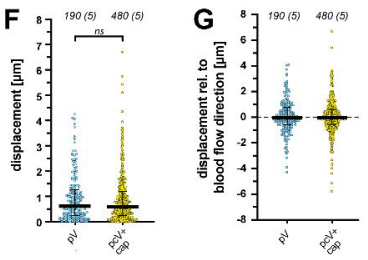

$\mathrm{x}$ [um]
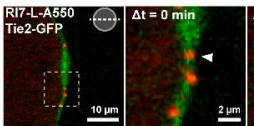

$10 \mu \mathrm{m}$

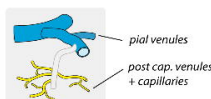

D
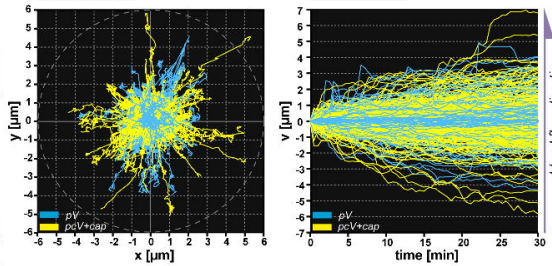

$\stackrel{y}{\longrightarrow} \rightarrow(x, y)$ coordinates blocd flow $v f$ movement rel to
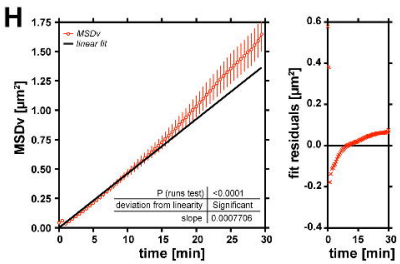

$\mathbf{K}$
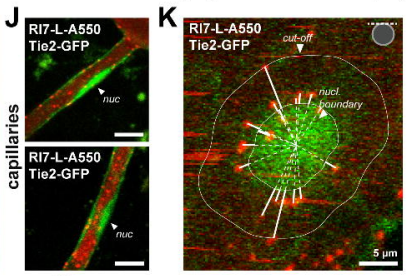

$\mathbf{N}$

RRI7-L-A550+Tie2-GFP

$n=3534$ (5)

- proximas

intermedlate

distal

non-classified

2.5-5.0 $\mathrm{mm}^{-} \quad 5.0-8.5 \mu \mathrm{m}$

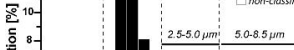

RI7-L-A550

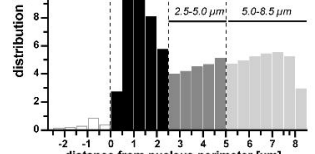




\section{A}

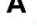

cap

R17-L-A550

Tie2-GFP

D

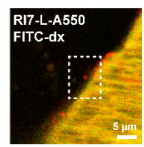

ven

R17-L-A550

Tie2-GFP
B

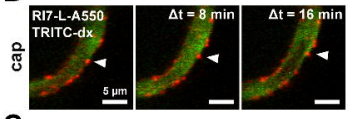

C
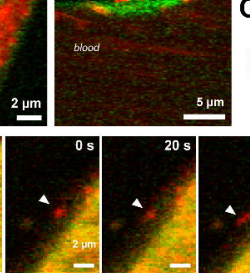

RI7-L-A550

C TRITC-dx

Ф

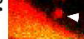

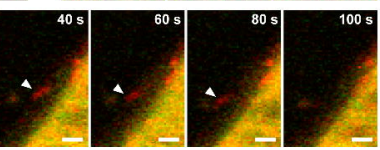

E
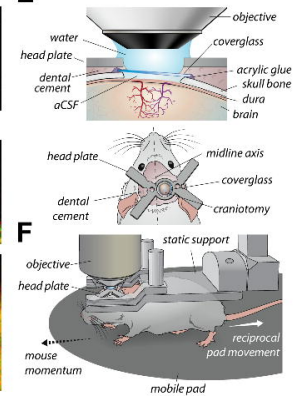

\begin{tabular}{c}
$\mathbf{G}$ \\
\\
$\frac{8}{3}$ \\
$\frac{8}{\$}$ \\
\hline
\end{tabular}

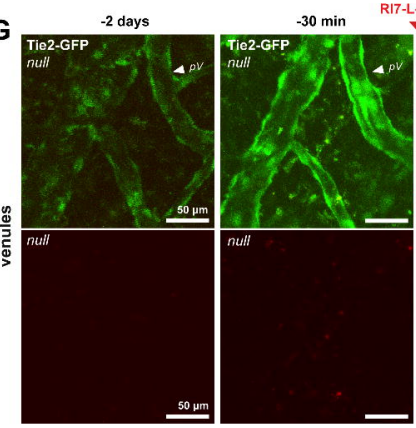

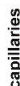

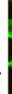

Tie2-GFP

nuh ${ }^{2}$

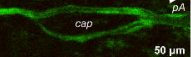

\section{Tie2-GFP}

null

$50 \mu \mathrm{m}$

2 days

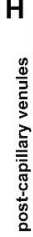

RI7-L-A550
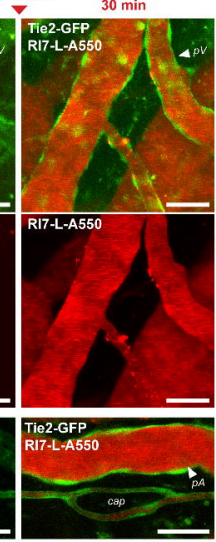

1 day
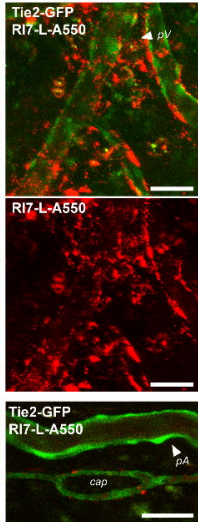

2 days
Tie2-GFP

Ri7-L-A550

R17-L-A550
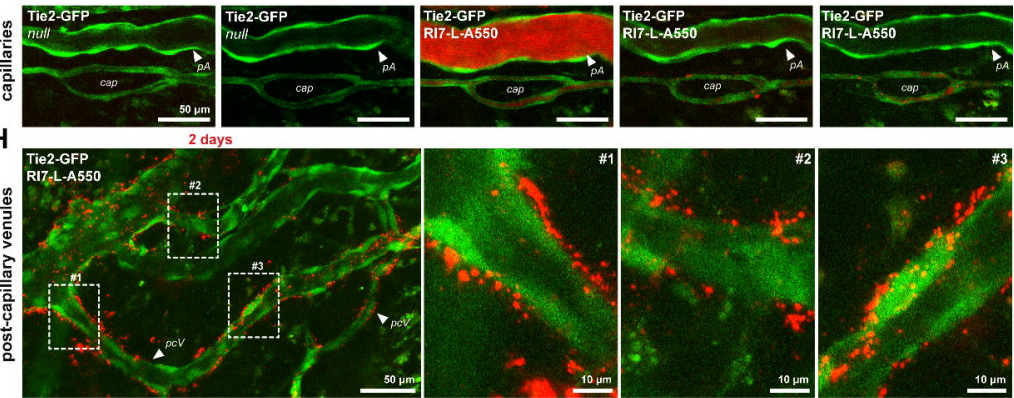


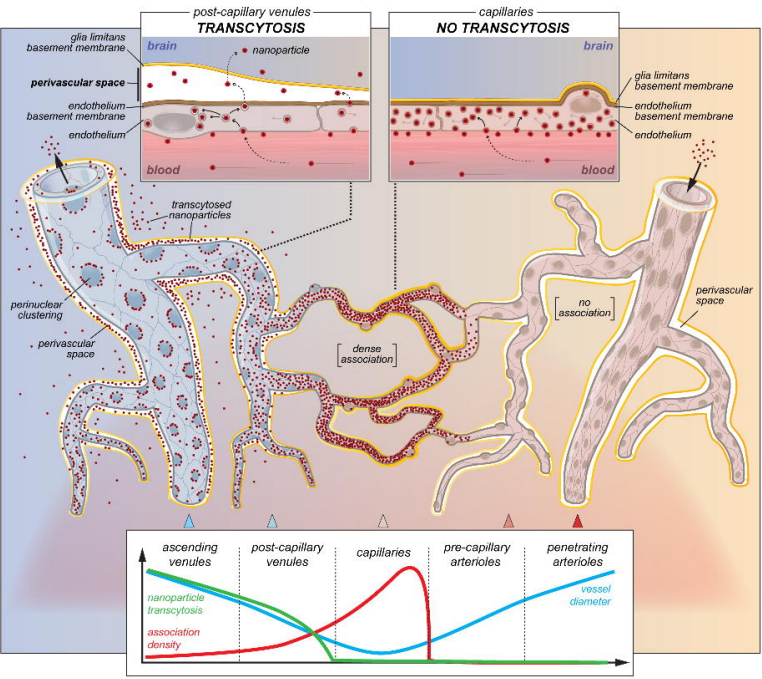

